\title{
Study of Galaxy Distributions with SDSS DR14 Data and Measurement of Neutrino Masses
}

\author{
B. Hoeneisen \\ Universidad San Francisco de Quito, Quito, Ecuador \\ Email: bhoeneisen@usfq.edu.ec
}

How to cite this paper: Hoeneisen, B. (2018) Study of Galaxy Distributions with SDSS DR14 Data and Measurement of Neutrino Masses. International Journal of Astronomy and Astrophysics, 8, 230-257. https://doi.org/10.4236/ijaa.2018.83017

Received: June 5, 2018

Accepted: August 20, 2018

Published: August 23, 2018

Copyright $\odot 2018$ by author and Scientific Research Publishing Inc. This work is licensed under the Creative Commons Attribution International License (CC BY 4.0).

http://creativecommons.org/licenses/by/4.0/

(c) (i) Open Access

\begin{abstract}
We study galaxy distributions with Sloan Digital Sky Survey SDSS DR14 data and with simulations searching for variables that can constrain neutrino masses. To be specific, we consider the scenario of three active neutrino eigenstates with approximately the same mass, so $\sum m_{v}=3 m_{v}$. Fitting the predictions of the $\Lambda$ CDM model to the Sachs-Wolfe effect, $\sigma_{8}$, the galaxy power spectrum $P_{\text {gal }}(k)$, fluctuations of galaxy counts in spheres of radii ranging from $16 / h$ to $128 / h \mathrm{Mpc}$, Baryon Acoustic Oscillation (BAO) measurements, and $h=0.678 \pm 0.009$, in various combinations, with free spectral index $n$, and free galaxy bias and galaxy bias slope, we obtain consistent measurements of $\sum m_{v}$. The results depend on $h$, so we have presented confidence contours
\end{abstract} in the $\left(\sum m_{v}, h\right)$ plane. A global fit with $h=0.678 \pm 0.009$ obtains $\sum m_{v}=0.719 \pm 0.312(\text { stat })_{-0.028}^{+0.055}$ (syst) $\mathrm{eV}$, and the amplitude and spectral index of the power spectrum of linear density fluctuations $P(k)$ :

$N^{2}=(2.09 \pm 0.33) \times 10^{-10}$, and $n=1.021 \pm 0.075$. The fit also returns the galaxy bias $b$ including its scale dependence.

\section{Keywords}

Neutrino Mass, Galaxy Power Spectrum

\section{Introduction}

We measure neutrino masses by comparing the predictions of the $\Lambda \mathrm{CDM}$ model with measurements of the power spectrum of linear density perturbations $P(k)$. We consider three measurements of $P(k): 1)$ the Sachs-Wolfe effect of fluctuations of the Cosmic Microwave Background (CMB) which is a direct measurement of density fluctuations [1] [2];2) the relative mass fluctuations $\sigma_{8}$ 
in randomly placed spheres of radius $r_{s}=8 / h \quad \mathrm{Mpc}$ with gravitational lensing and studies of rich galaxy clusters [2] [3]; and 3) measurements of $P(k)$ inferred from galaxy clustering with the Sloan Digital Sky Survey [4] [5] [6]. Baryon Acoustic Oscillations (BAO) were considered separately [7] [8] and are not included in the present study, except for the final combinations.

To be specific, we consider three active neutrino eigenstates with nearly the same mass, so $\sum m_{v}=3 m_{v}$. This is a useful scenario to consider because the current limits on $m_{v}^{2}$ are much larger than the mass-squared-differences $\Delta m^{2}$ and $\Delta m_{21}^{2}$ obtained from neutrino oscillations [3].

Figures 1-4 illustrate the problem at hand. Figures 1-3 present measurements of the "reconstructed" galaxy power spectrum $P_{\text {gal }}(k)$ obtained from the SDSS-III BOSS survey [4], while Figure 4 presents the corresponding "standard" $P_{\text {gal }}(k)$. The "reconstructed" $P_{\text {gal }}(k)$ is obtained from the directly measured "standard" $P_{\mathrm{gal}}(k)$ by subtracting peculiar motions to obtain the power spectrum prior to non-linear clustering. Also shown are various fits to this data (with floating normalization), and to measurements of the Sachs-Wolfe effect, and $\sigma_{8}$. The Sachs-Wolfe effect normalizes $P(k)$, within its uncertainty, in the approximate range of $\log _{10}\left(k /\left(h \mathrm{Mpc}^{-1}\right)\right)$ from -3.1 to -2.7 , while $\sigma_{8}$ is most sensitive to the range -1.3 to -0.6 . Full details will be given in the main body of this article.

The fit in Figure 1 corresponds to the function

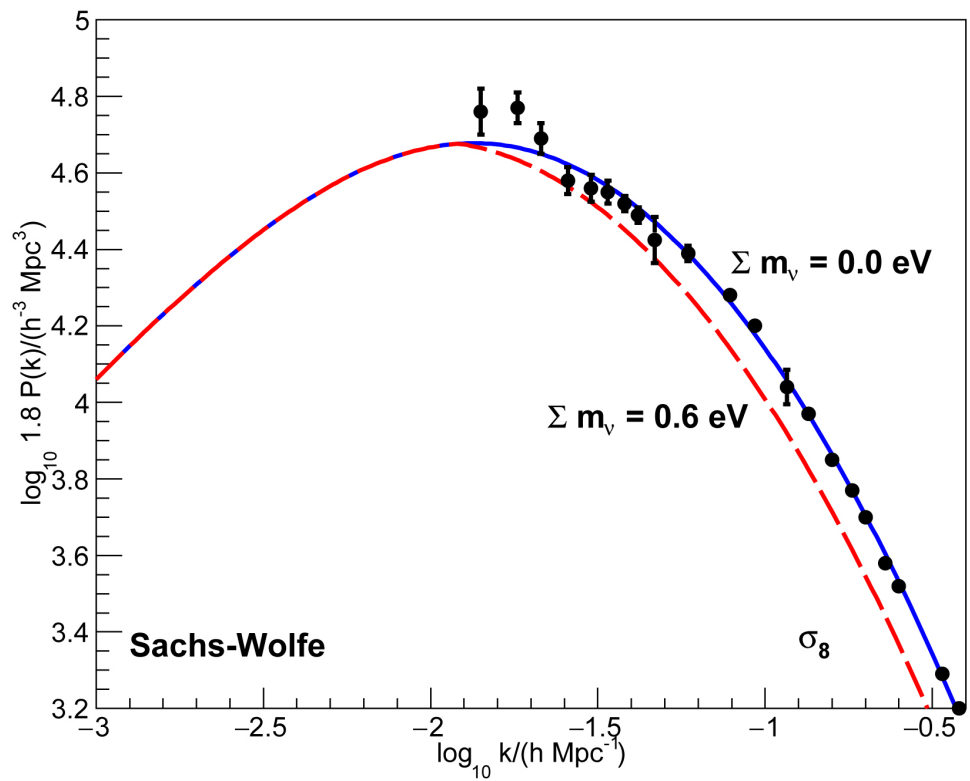

Figure 1. Comparison of $P_{\text {gal }}(k)$ obtained from the SDSS-III BOSS survey [4] ("reconstructed") with $b^{2} P(k)$ obtained from a fit of Equation (5) with $\sum m_{v}=0 \mathrm{eV}$ to the Sachs-Wolfe effect, $\sigma_{8}$, and $P_{\text {gal }}(k)$. The fit obtains $A=8738 \mathrm{Mpc}^{3}$, $k_{\mathrm{eq}}=0.068 h \mathrm{Mpc}^{-1}, \eta=4.5$, and $b^{2}=1.8$, with $\chi^{2}=24.7$ for 19 degrees of freedom. Also shown for comparison is the curve with the same parameters, except $\sum m_{v}=0.6$ $\mathrm{eV}$. 


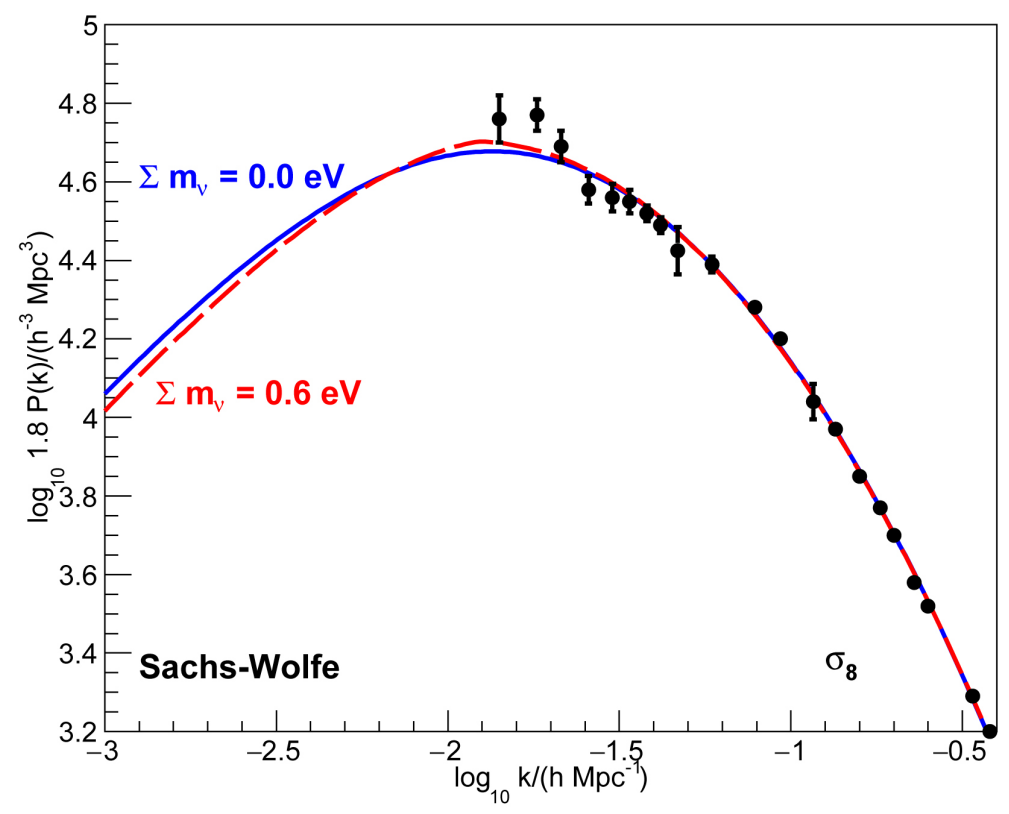

Figure 2. Same as Figure 1, except that the curve " $\sum m_{v}=0.6 \mathrm{eV}$ " is fit. We obtain $A=9312 \mathrm{Mpc}^{3}, k_{\text {eq }}=0.080 h \mathrm{Mpc}^{-1}, \eta=4.2$, and $\kappa=1.8$, with $\chi^{2}=21.8$ for 19 degrees of freedom. Note that $\sum m_{v}$ is largely degenerate with the remaining parameters in Equation (5), unless we are able to constrain $k_{\text {eq }}$.



Figure 3. Comparison of $P_{\text {gal }}(k)$ obtained from the SDSS-III BOSS survey [4] ("reconstructed") with $b^{2} P(k)$ obtained from a fit of Equation (6) to the Sachs-Wolfe effect, $\sigma_{8}$, and $P_{\text {gal }}(k)$. The fit obtains $\sum m_{v}=0.014 \pm 0.079 \mathrm{eV}, N^{2}=(1.41 \pm 0.12) \times 10^{-10}$, and $b^{2}=1.7 \pm 0.1$, with $\chi^{2}=47$ for 20 degrees of freedom (so the statistical uncertainties shown need to be multiplied by $\sqrt{47 / 20}$ ). Also shown is the fit with $\sum m_{v}=0.6 \mathrm{eV}$ fixed for comparison. 


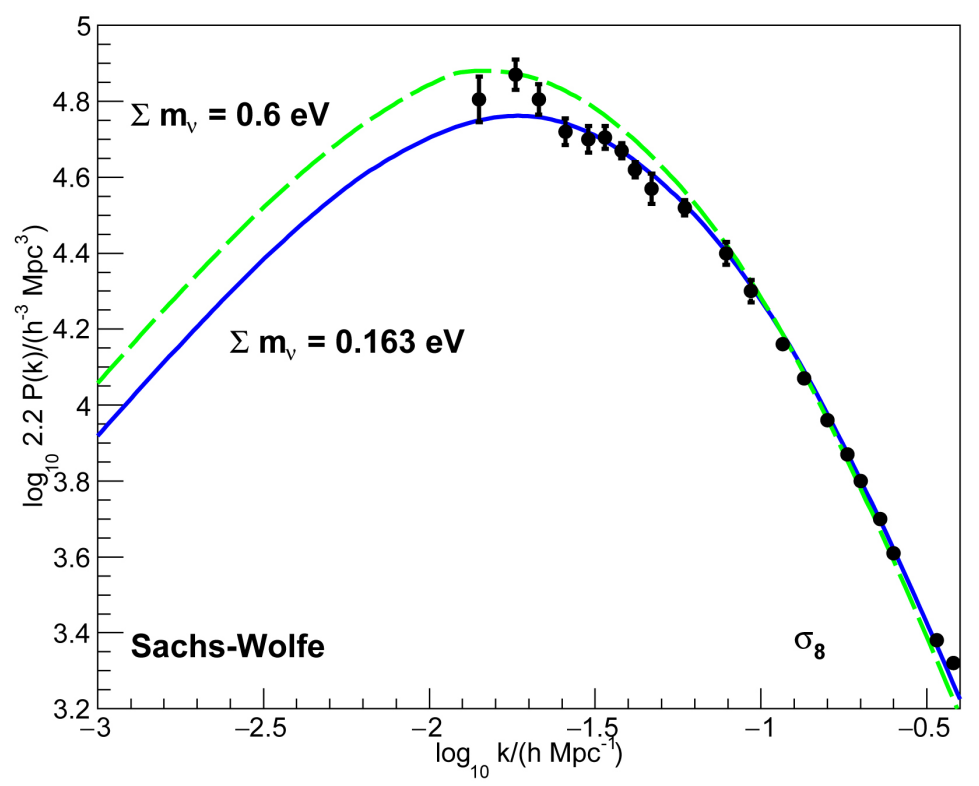

Figure 4. Comparison of $P_{\text {gal }}(k)$ obtained from the SDSS-III BOSS survey [4] ("standard") with $b^{2} P(k)$ obtained from a fit of Equation (6) to the Sachs-Wolfe effect, $\sigma_{8}$, and $P_{\text {gal }}(k)$. The fit obtains $\sum m_{v}=0.163 \pm 0.061 \mathrm{eV}, N^{2}=(1.56 \pm 0.12) \times 10^{-10}$, and $b^{2}=2.2 \pm 0.2$, with $\chi^{2}=33.9$ for 20 degrees of freedom (so the statistical uncertainties shown need to be multiplied by $\sqrt{33.9 / 20}$ ). Also shown is the fit with $\sum m_{v}=0.6 \mathrm{eV}$ fixed for comparison.

$$
P^{\prime}(k) \equiv \frac{(2 \pi)^{3} a_{20}^{2} A w^{n}}{\left(1+\eta w+w^{2}\right)^{2}},
$$

where $w \equiv k / k_{\mathrm{eq}}$. Unless otherwise noted, we take the Harrison-Zel'dovich index $n=1$ which is close to observations. The parameters $A, \eta$, and $k_{\text {eq }}$, as well as the normalization factor $b^{2}$, are free in the fit. The uncertainties of two data points that fall on $\mathrm{BAO}$ peaks are multiplied by three (since BAO is not included in $\left.P^{\prime}(k)\right)$.

Also shown in Figure 1 is the suppression of $P(k)$ for $k$ greater than

$$
k_{\mathrm{nr}}=0.018 \cdot \Omega_{m}^{1 / 2}\left(\frac{\sum m_{v}}{1 \mathrm{eV}}\right)^{1 / 2} h \mathrm{Mpc}^{-1}
$$

due to free-streaming of massive neutrinos that can not cluster on these small scales, and, more importantly, to the slower growth of structure with massive neutrinos [9]. The suppression factor for $k \gg k_{\mathrm{nr}}$ for one massive neutrino, or three degenerate massive neutrinos, is

$$
f\left(k, \sum m_{v}\right) \equiv \frac{P(k)^{f_{v}}}{P(k)^{f_{v}=0}}=1-8 f_{v},
$$

where $f_{v}=\Omega_{v} / \Omega_{m}$ [9]. $\Omega_{m}$ is the total (dark plus baryonic plus neutrino) matter density today relative to the critical density, and includes the contribu- 
tion $\Omega_{v}$ of neutrinos that are non-relativistic today. $\Omega_{v}=h^{-2} \sum m_{v} / 93.04 \mathrm{eV}$ for three left-handed plus right-handed Majorana neutrino eigenstates, or three eigenstates of left-handed Dirac neutrinos plus three right-handed Dirac anti-neutrinos, that are non-relativistic today (right-handed Dirac neutrinos and left-handed Dirac anti-neutrinos are assumed to not have reached thermal and chemical equilibrium with the Standard Model particles). We take $f\left(k, \sum m_{v}\right)=1$ for $k<k_{\mathrm{nr}} / 0.604$, and

$$
f\left(k, \sum m_{v}\right)=1-0.407 \frac{\sum m_{v}}{0.6 \mathrm{eV}}\left[1-\left(\frac{k_{\mathrm{nr}}}{0.604 k}\right)^{0.494}\right]
$$

for $k>k_{\text {nr }} / 0.604$ and $\sum m_{v}<1.1 \mathrm{eV}$, for galaxy formation at a redshift $z=0.5$ [9].

Figure 2 is the same as Figure 1 except that the function

$$
P(k)=P^{\prime}(k) f\left(k, \sum m_{v}\right)
$$

with $\sum m_{v}=0.6 \mathrm{eV}$ is fit. We see that the parameter $\sum m_{v}$ is largely degenerate with the parameters $A, \eta$, and $k_{\text {eq }}$, so that only a weak sensitivity to $\sum m_{v}$ is obtained unless we are able to constrain $k_{\text {eq }}$. The power spectrum $P^{\prime}(k)$ of Equation (1) neglects the growth of structure inside the horizon while radiation dominates.

The fits in Figure 3 and Figure 4 make full use of the $\Lambda$ CDM theory. The fitted function is

$$
P(k)=P^{\prime \prime}(k) f\left(k, \sum m_{v}\right),
$$

where $P^{\prime \prime}(k)$ is given by [2]:

$$
P^{\prime \prime}(k)=\frac{4(2 \pi)^{3} N^{2} C^{2} k \tau^{2}\left(\sqrt{2} k / k_{\mathrm{eq}}\right)}{25 \Omega_{m}^{2} H_{0}^{4}}\left(\frac{k_{\mathrm{SW}}}{k}\right)^{1-n},
$$

with

$$
k_{\mathrm{eq}}=\frac{\sqrt{2} H_{0}\left(\Omega_{m}-\Omega_{v}\right)}{\sqrt{\Omega_{r}}} .
$$

$C$ is a function of $\Omega_{\Lambda} / \Omega_{m}$, and we take $C=0.767$ [2]. $\tau\left(\sqrt{2} k / k_{\mathrm{eq}}\right)$ is a function given in Reference [2]. The pivot point $k_{\mathrm{SW}}=0.001 \mathrm{Mpc}^{-1}$ is chosen to not upset Equation (41) below. The fit depends on $h, \Omega_{m}$, and the spectral index $n$, so we define $\delta h \equiv(h-0.678) / 0.009 \quad[3], \delta \Omega_{m} \equiv\left(\Omega_{m}-0.281\right) / 0.003 \quad$ [7], and $\delta n \equiv(n-1) / 0.038 \quad$ [3], and obtain, tentatively,

$$
\begin{aligned}
\sum m_{v}= & 0.014+0.162 \cdot \delta h+0.807 \cdot \delta n+0.142 \cdot \delta \Omega_{m} \\
& \pm 0.079 \sqrt{47 / 20}(\text { stat })_{-0.007}^{+0.005}(\text { syst }) \mathrm{eV},
\end{aligned}
$$

by minimizing the $\chi^{2}$ with respect to $\sum m_{v}$, and $N^{2}$. The statistical uncertainty has been multiplied by the square root of the $\chi^{2}$ per degree of freedom. This result corresponds to the "reconstructed" data in Figure 3. The systematic uncertainties included are from the top-hat window function instead of the 
gaussian window function, and an alternative value of $\sigma_{8}$ (details will be given in Section 3). Not included is the systematic uncertainty due to the possible scale dependence of the galaxy bias $b$.

To obtain $P(k)$, we would like to measure the density $\rho(\boldsymbol{r}, z)$ at redshift $z$, but we only have information on the peaks of $\rho(\boldsymbol{r}, z)$ that have gone non-linear collapsing into visible galaxies. How accurate is the measurement of $P(k)$ with galaxies? The measurement of $P_{\text {gal }}(k)$ in Ref. [4] is based on a procedure described in [10] based on "the usual assumption that the galaxies form a Poisson sample [11] of the density field". In other words, the assumption is that the number density of point galaxies $n(\mathbf{x})$ is equal to its expected mean $\bar{n}$ (which depends on the position dependent galaxy selection criteria), modulated by the perturbation of the density field:

$$
\frac{n(\boldsymbol{x})}{\bar{n}}=b \frac{\rho(\boldsymbol{x})}{\bar{\rho}} \equiv b\left(1+\delta_{c}(\boldsymbol{x})\right) .
$$

Both sides of this equation are measured or calculated at the same length scale, and at the same time. The "galaxy bias" $b$ is explicitly assumed to be scale invariant. If we choose a region of space such that $\bar{n}$ is constant, then the galaxy power spectrum $P_{\text {gal }}(k)$ (derived from $n(x) / \bar{n}$ ) should be proportional, under the above assumption, to the power spectrum of linear density perturbations $P(k)$ (derived from $\left.\delta_{c}(\boldsymbol{x})\right)$ up to corrections:

$$
P_{\text {gal }}(k)=b^{2} P(k) \text {. }
$$

It is due to this bias $b$ that we have freed the normalization of the measured $P_{\text {gal }}(k)$ in the fits corresponding to Figures 1-4.

In the following Sections we study galaxy distributions with SDSS DR14 data and with simulations, in order to understand their connection with the underlying power spectrum of linear density fluctuations $P(k)$. In the end we return to the measurement of neutrino masses.

\section{The Hierarchical Formation of Galaxies}

This Section allows a precise definition of $P(k)$, and an understanding of the connection between $P(k)$ and $P_{\text {gal }}(k)$. We generate galaxies as follows (see [12] for full details). The evolution of the Universe in the homogeneous approximation is described by the Friedmann equation

$$
\frac{1}{H_{0}} \frac{1}{a_{1}} \frac{\mathrm{d} a_{1}}{\mathrm{~d} t} \equiv E\left(a_{1}\right)=\sqrt{\frac{\Omega_{r}}{a_{1}^{4}}+\frac{\Omega_{m}}{a_{1}^{3}}+\frac{\Omega_{k}}{a_{1}^{2}}+\Omega_{\Lambda}} .
$$

The expansion parameter $a_{1}(t)$ has been normalized to 1 at the present time $t_{0}: a_{1}\left(t_{0}\right)=1 . H_{0}$ has been normalized so $E(1)=1$. Therefore $H_{0}$ is the present Hubble expansion rate. With these normalizations we have $\Omega_{r}+\Omega_{m}+\Omega_{k}+\Omega_{\Lambda} \equiv 1$. The matter density is $\rho_{m}(t)=\Omega_{m} \rho_{c} / a_{1}^{3}$, where $\rho_{c}=3 H_{0}^{2} /\left(8 \pi G_{N}\right)$ is the critical density of the Universe. We are interested in the period after the density of matter exceeds the density of radiation. For our simulations we assume flat space, i.e. $\Omega_{k}=0$, we neglect the radiation density 
$\Omega_{r}$, take $\Omega_{\Lambda}=0.719$ constant [7], and the present Hubble expansion rate $H_{0}=100 h \mathrm{~km} \cdot \mathrm{s}^{-1} \cdot \mathrm{Mpc}^{-1}$ with $h=0.678$ [3]. The solution to Equation (12) with these parameters is shown by the curve " $a_{1}$ " in Figure 5 . The present age of the universe with these parameters is $t_{0}=14.1 \mathrm{Gyr}$.

Setting $\Omega_{\Lambda}=0$ we obtain the critical universe with expansion parameter

$$
a_{2}=\left[\frac{3 H_{0}}{2}\right]^{2 / 3} \Omega_{m}^{1 / 3} t^{2 / 3}
$$

also shown in Figure 5. We note that $a_{2}\left(t_{0}\right) \equiv a_{20}=0.846$. Let us now add density fluctuations to this critical universe and consider a density peak. The growing mode for this density peak is obtained by adding a negative $\Omega_{k}$ to the critical Universe. This prescription is exact if the density peak is spherically symmetric. An example with "expansion parameter" $a_{4}$ is presented in Figure 5. Note that $a_{4}$ grows to maximum expansion and then collapses to zero at time $t_{4}=\pi \Omega_{m} /\left[\left(-\Omega_{k}\right)^{3 / 2} H_{0}\right]$, and, in our model [12], a galaxy forms. In the example of Figure 5 the galaxy forms at redshift $z=0.5 \cdot a_{3}(t)$ is the linear approximation to $a_{4}(t)$. In the linear approximation for growing modes the density fluctuations relative to $\rho_{2}$ grow in proportion to $a_{2}(t)$ :

$$
\delta_{c} \equiv \frac{\rho_{3}-\rho_{2}}{\rho_{2}}=3 \frac{a_{2}-a_{3}}{a_{2}}=-\frac{3 \Omega_{k}}{5 \Omega_{m}} a_{2}(t),
$$

while $\delta_{c} \ll 1$. At the time $t_{4}$, when the galaxy forms, $\delta_{c} \equiv\left(\rho_{3}-\rho_{2}\right) / \rho_{2}=1.69$ in the linear approximation (which has already broken down).

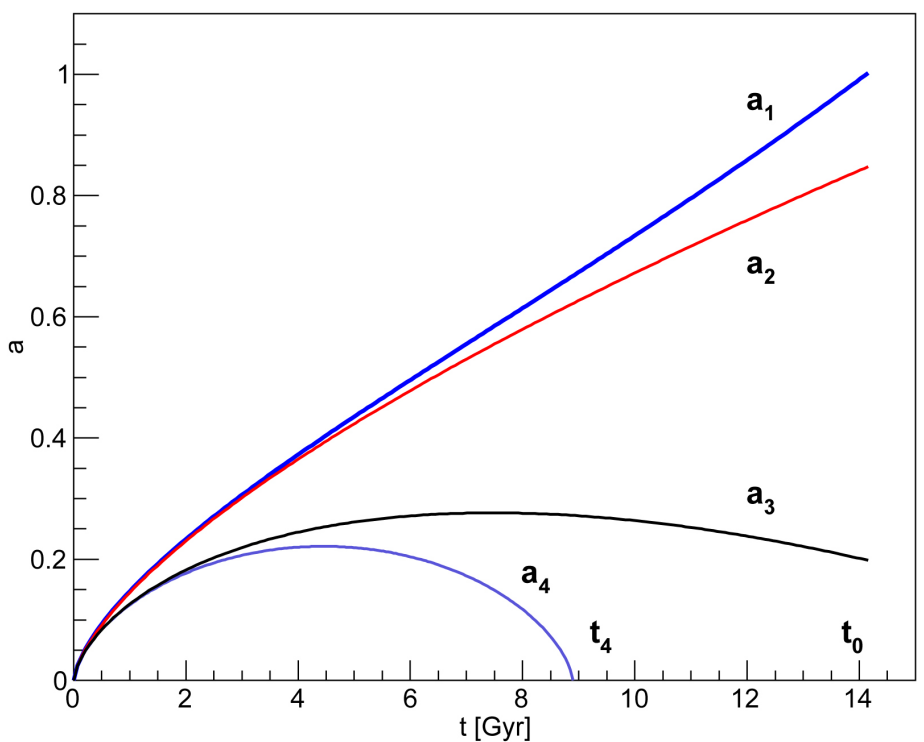

Figure 5. Expansion parameters as a function of time $t$ of four solutions of the Friedmann equation. From top to bottom, $a_{1}$ corresponds to $\left(\Omega_{m}, \Omega_{\Lambda}, \Omega_{k}\right)=(0.281,0.719,0), a_{2}$ corresponds to $(0.281,0,0), a_{3}$ is the linear approximation to $(0.281,0,-1.27)$, while $a_{4}$ is the exact solution to $(0.281,0,-1.27) \cdot a_{4}$ is the exact solution for the growing mode of a spherically symmetric density peak that collapses to a galaxy at $t_{4}$. In all cases $h=0.678$. 
In the linear approximation the density due to Fourier components of wavevector $|\boldsymbol{k}| \leq k_{I}$ is

$$
\rho_{\text {lin }}(\boldsymbol{x}, t, I)=\frac{\Omega_{m} \rho_{c}}{a_{2}^{3}}\left\{1+\delta_{c}(\boldsymbol{x}, t, I)\right\},
$$

where

$$
\delta_{c}(\boldsymbol{x}, t, I)=\sum_{\boldsymbol{k}, k \leq k_{I}}\left|\delta_{\boldsymbol{k}}\right| a_{2}(t) \exp \left[i \frac{\boldsymbol{k} \cdot \boldsymbol{x}}{a_{2}(t)}+i \varphi_{\boldsymbol{k}}\right] .
$$

$\varphi_{k}$ are random phases. The sum of the Fourier series is over comoving wavevectors that satisfy periodic boundary conditions in a rectangular box of volume $V=L_{x} L_{y} L_{z}$ :

$$
\boldsymbol{k}=2 \pi\left(\frac{n}{L_{x}} \hat{i}+\frac{m}{L_{y}} \hat{j}+\frac{l}{L_{z}} \hat{k}\right),
$$

where $L_{x}=n_{\text {max }} L_{0}, \quad L_{y}=m_{\max } L_{0}, L_{z}=l_{\max } L_{0}, n, m, l=0, \pm 1, \pm 2, \pm 3, \cdots$, and

$$
k_{I}=k_{\max } \frac{I}{I_{\max }}
$$

where $k_{\max }=2 \pi / L_{0}$, and $I=1,2, \cdots, I_{\max }$.

Inverting Equation (16) obtains

$$
\delta_{\boldsymbol{k}} \equiv\left|\delta_{\boldsymbol{k}}\right| \mathrm{e}^{i \varphi_{\boldsymbol{k}}}=\delta_{-\boldsymbol{k}}^{*}=\frac{1}{V} \int_{V} \frac{\delta_{c}(\boldsymbol{x}, t)}{a_{2}(t)} \exp (-i \boldsymbol{k} \cdot \boldsymbol{X}) \mathrm{d}^{3} \boldsymbol{X},
$$

where $\boldsymbol{X} \equiv \boldsymbol{x}(t) / a_{2}(t)$ is the comoving coordinate in the linear approximation. The power spectrum of density fluctuations

$$
P(\boldsymbol{k}) \equiv V\left|\delta_{k}\right|^{2} a_{20}^{2}
$$

is defined in the linear approximation corresponding to $a_{3}$, and is approximately independent of $V$ for large $V$. Averaging over $\boldsymbol{k}$ in a bin of $k \equiv|\boldsymbol{k}|$ obtains $P(k)$. Note that

$$
\frac{1}{V} \sum_{\boldsymbol{k}} P(\boldsymbol{k})=a_{20}^{2} \sum_{\boldsymbol{k}}\left|\delta_{\boldsymbol{k}}\right|^{2}=\frac{1}{V} \int_{V} \delta_{c}^{2}\left(\boldsymbol{x}, t_{0}\right) \mathrm{d}^{3} \boldsymbol{X} .
$$

Each term in this equation is approximately independent of $V$. The Fourier transform of the power spectrum is the correlation function:

$$
\sum_{\boldsymbol{k}} P(\boldsymbol{k}) \mathrm{e}^{i \boldsymbol{k} \cdot \boldsymbol{X}}=\int_{V} \delta_{c}\left(\boldsymbol{x}^{\prime}, t_{0}\right) \delta_{c}\left(\boldsymbol{x}^{\prime}+\boldsymbol{x}, t_{0}\right) \mathrm{d}^{3} \boldsymbol{X}^{\prime} .
$$

The generation of galaxies at time $t$ proceeds as follows. We start with $I=2$, calculate $\delta_{c}(\boldsymbol{x}, t, I)$, and search for local maximums of $\delta_{c}(\boldsymbol{x}, t, I)$ inside a comoving volume $L_{x} L_{y} L_{z}$. If a maximum exceeds 1.69 we generate a galaxy of radius

$$
R(I) \approx \frac{\pi a_{2}}{k_{I}},
$$

and dark plus baryonic plus neutrino mass 


$$
M(I) \approx 2.69 \frac{4 \pi R(I)^{3}}{3} \frac{\Omega_{m} \rho_{c}}{a_{2}^{3}}
$$

if it "fits", i.e. if it does not overlap previously generated galaxies. $I$ is increased by 1 unit to generate galaxies of a smaller generation, until $I=I_{\max }$ is reached. See Figure 6.

The peculiar velocity of the generated galaxies is

$$
\boldsymbol{v}_{\mathrm{pec}}(\boldsymbol{x}, t)=\sum_{\boldsymbol{k}, k<k_{I-1}} \frac{2 i \boldsymbol{k} a_{2}^{2}\left|\delta_{\boldsymbol{k}}\right|}{3 k^{2} t} \exp \left[i \frac{\boldsymbol{k} \cdot \boldsymbol{x}}{a_{2}}+i \varphi_{\boldsymbol{k}}\right],
$$

and their peculiar displacement is

$$
\boldsymbol{x}_{\mathrm{pec}}=\frac{3}{2} \boldsymbol{v}_{\mathrm{pec}} t .
$$

$\boldsymbol{x}+\boldsymbol{x}_{\mathrm{pec}}$ is the proper coordinate of a galaxy at the time $t$ of its generation, and $a_{2} \equiv a_{2}(t)$. The comoving coordinate of this galaxy, i.e. its position extrapolated to the present time, is the corresponding $\left(\boldsymbol{x}+\boldsymbol{x}_{\mathrm{pec}}\right) / a_{1}(t) . \boldsymbol{x}_{\mathrm{pec}}$ causes the difference between the data points $P_{\text {gal }}(k)$ in Figure 3 and Figure 4 at large $k$.

Note in Figure 6 that the formation of galaxies is hierarchical: small galaxies form first, and, as time goes on, density perturbations grow, and groups of galaxies coalesce into larger galaxies in an ongoing process until dark energy dominates and the hierarchical formation of galaxies comes to an end. The distribution of galaxies of generation $I$ depend only on $P(k)$ for $k<k_{I}$. Also,

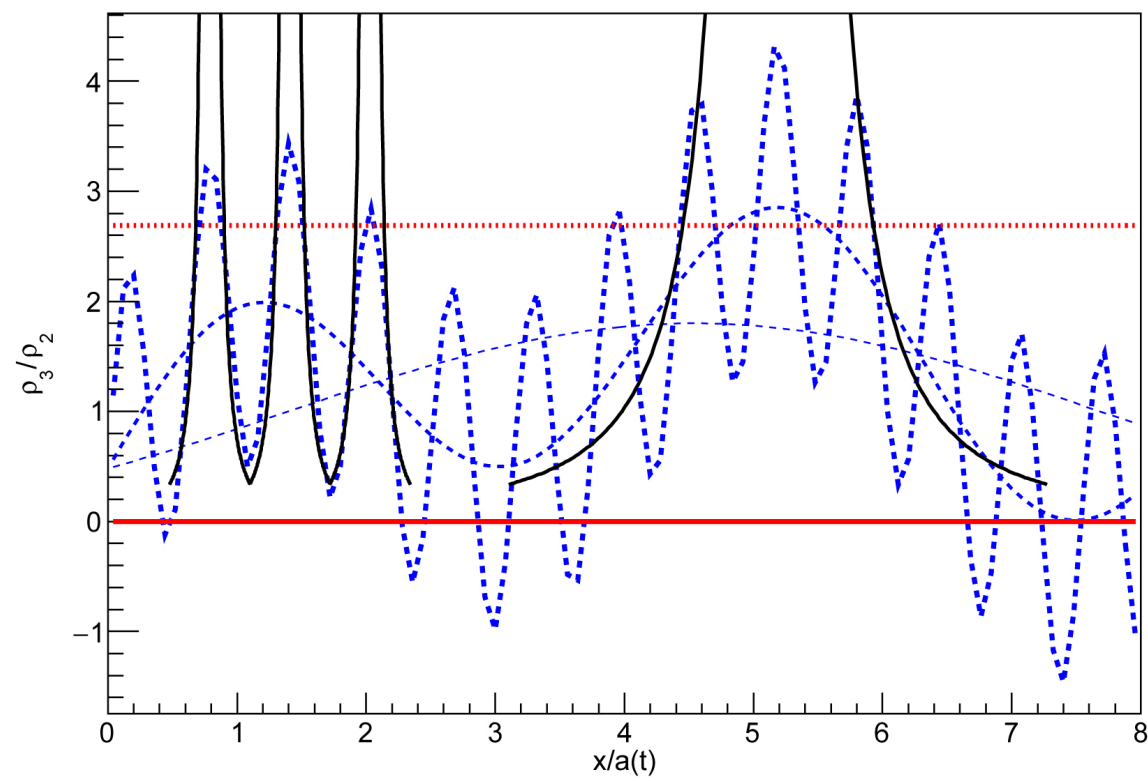

Figure 6. The hierarchical formation of galaxies [12]. Three Fourier components of the density in the linear approximation are shown. Note that in the linear approximation $\delta_{c} \equiv\left(\rho_{3}-\rho_{2}\right) / \rho_{2} \propto a(t)$. When $\delta_{c}$ reaches 1.69 in the linear approximation the exact solution diverges and a galaxy forms. As time goes on, density perturbations grow, and groups of galaxies of one generation coalesce into larger galaxies of a new generation as shown on the right. 
luminous galaxies occupy a total volume (luminous plus dark) less than 1/2.69 of space.

Neutrinos with $0<m_{v}<1.17 \mathrm{eV}$ become non-relativistic after the densities of radiation and matter become equal, as illustrated in Figure 7.

\section{Fluctuation Amplitude $\sigma_{8}$}

$\sigma_{8}$ is the root-mean-square fluctuation of total mass relative to the mean in randomly placed volumes of radius $r_{s}=8 h^{-1} \mathrm{Mpc}$. We use a "gaussian window function"

$$
W(r)=\frac{1}{V_{W}} \exp \left(-\frac{r^{2}}{2 r_{W}^{2}}\right)
$$

which smoothly defines a volume

$$
V_{W} \equiv \frac{4}{3} \pi r_{s}^{3}=(2 \pi)^{3 / 2} r_{W}^{3} .
$$

Note that

$$
\int_{0}^{\infty} W(r) 4 \pi r^{2} \mathrm{~d} r=1 .
$$

The Fourier transform of $W(r)$ is

$$
W(k) \equiv \int W(r) \mathrm{e}^{-i k \cdot r} \mathrm{~d}^{3} r=\exp \left(-k^{2} r_{W}^{2} / 2\right) .
$$

Then



Figure 7. Example with $m_{v}=0.46 \mathrm{eV}$ for each of 3 active neutrino eigenstates. Neutrinos become non-relativistic at $a_{v} \approx 0.00115$. The matter density relative to the critical density is $\Omega_{m}-\Omega_{v}$ for $a<a_{v}$, and $\Omega_{m}$ for $a>a_{v}$. The densities of matter and radiation become equal at $a_{\mathrm{eq}}=0.00036$. 


$$
\sigma_{8}^{2}=\frac{1}{(2 \pi)^{3}} \int_{0}^{\infty} 4 \pi k^{2} \mathrm{~d} k P(k) \exp \left(-k^{2} r_{W}^{2}\right) .
$$

An alternative window function is the "top hat" function $f(r)=3 /\left(4 \pi r_{s}^{3}\right)$ for $r<r_{s}$, and $f(r)=0$ for $r>r_{s}$. Then

$$
f(k)=\frac{3}{\left(k r_{s}\right)^{3}}\left(\sin \left(k r_{s}\right)-k r_{s} \cos \left(k r_{s}\right)\right) .
$$

Direct measurements obtain [3]

$$
\sigma_{8}=[0.813 \pm 0.013 \text { (stat) } \pm 0.024(\text { syst })]\left(\Omega_{m} / 0.25\right)^{-0.47} .
$$

$80 \%$ of $\sigma_{8}^{2}$ is due to $k / h$ in the range 0.05 to $0.25 \mathrm{Mpc}^{-1}$. For comparison, from the 6-parameter $\Lambda \mathrm{CDM}$ fit [3], $\sigma_{8}=0.815 \pm 0.009$.

\section{The Sachs-Wolfe Effect}

The spherical harmonic expansion of the $\mathrm{CMB}$ temperature fluctuation is

$$
\Delta T(\theta, \phi) \equiv T(\theta, \phi)-T_{0}=\sum_{l m} a_{l m} Y_{l m}(\theta, \phi) .
$$

Averaging over $m$ obtains $C_{l} \equiv\left\langle\left|a_{l m}\right|^{2}\right\rangle$. The variable that is measured is [2]

$$
\left\langle\Delta T\left(\hat{n}_{1}\right) \Delta T\left(\hat{n}_{2}\right)\right\rangle=\sum_{l} C_{l} \frac{2 l+1}{4 \pi} P_{l}\left(\hat{n}_{1} \cdot \hat{n}_{2}\right) .
$$

For $7<l<20$ the dominant contribution to $C_{l}$ is from the Sachs-Wolfe effect [1] [2] [3]. This range corresponds to $0.0007 \mathrm{Mpc}^{-1}<k / h<0.002 \mathrm{Mpc}^{-1}$. The Sachs-Wolfe effect relates temperature fluctuations of the CMB to perturbations of the gravitational potential $\phi$ [2]:

$$
\left(\frac{\Delta T(\hat{n})}{T_{0}}\right)_{\mathrm{SW}}=\frac{1}{3} \delta \phi(\hat{n}) .
$$

When expressed as a function of comoving coordinates, $\delta \phi(\boldsymbol{X})$ is independent of time when matter dominates. The primordial power spectrum of gravitational potential fluctuations is assumed to have the form [2]

$$
P_{\phi}(k)=N_{\phi}^{2} k^{n-4} .
$$

The relation between $N_{\phi}^{2}$ and $N^{2}$ is $N_{\phi}^{2}=9 N^{2} / 25$ [2]. In the present analysis, unless otherwise stated, we assume the Harrison-Zel'dovich power spectrum with $n=1$, which is close to observations [3]. For $7 \lesssim l \lesssim 20$, [2]

$$
C_{l}=\frac{24 \pi Q^{2}}{5 l(l+1)},
$$

where the "quadrupole moment" $Q$ is measured to be

$$
Q=18.0 \pm 1.4 \mu \mathrm{K}
$$

from the 1996 COBE results (see list of references in [2]). Then, for $P^{\prime}(k)$,

$$
\frac{A}{k_{\text {eq }}}=\frac{1}{\Omega_{m}^{2}}\left(\frac{c}{H_{0}}\right)^{4} \frac{12 Q^{2}}{5 \pi T_{0}^{2}}=(16 \pm 3) \times 10^{4} \mathrm{Mpc}^{4},
$$


and for $P^{\prime \prime}(k)$,

$$
N^{2}=\frac{15}{\pi} \frac{Q^{2}}{T_{0}^{2}}=(2.08 \pm 0.33) \times 10^{-10},
$$

independently of $\sum m_{v}$. Detailed integration obtains results within $10 \%$ for $5<l<18$.

\section{Data and Simulations}

The data are obtained from the publicly available SDSS DR14 catalog [5] [6], see acknowledgement. We consider objects classified as GALAXY, with redshift $z$ in the range 0.4 to 0.6 , with redshift error $z E r r<0.002$, passing quality selection flags. We further select galaxies in the northern galactic cap, in a "rectangular" volume with $L_{x}=400 \mathrm{Mpc}$ along the line of sight (corresponding to redshift $z \approx 0.5 \pm 0.046), \quad L_{y}=3800 \mathrm{Mpc}$ (corresponding to an angle $86^{\circ}$ across the sky), and $L_{z}=1400 \mathrm{Mpc}$ (corresponding to an angle $32^{\circ}$ ). In total 222470 galaxies pass these selections. The distributions of these galaxies are shown in Figure 8.

Unless otherwise specified, the simulations have $L_{x}=L_{y}=L_{z}=700 \mathrm{Mpc}$, $I_{\max }=59, h=0.678, \Omega_{\Lambda}=0.719, \Omega_{m}=0.281, \Omega_{k}=0$, and the input power spectrum of density fluctuations is (5) with $A=9200 \mathrm{Mpc}^{3}, k_{\text {eq }} / h=0.067$ $\mathrm{Mpc}^{-1}, \quad \eta=4.7$, and $\sum m_{v}=0 \mathrm{eV}$. We generate galaxies at redshift $z=a_{1}^{-1}-1=0.5$, corresponding to $t=8.9 \mathrm{Gyr}$, and $a_{2}(t)=0.62$. This reference simulation has 34,444 galaxies, which is near the limit we can generate with available computing resources.

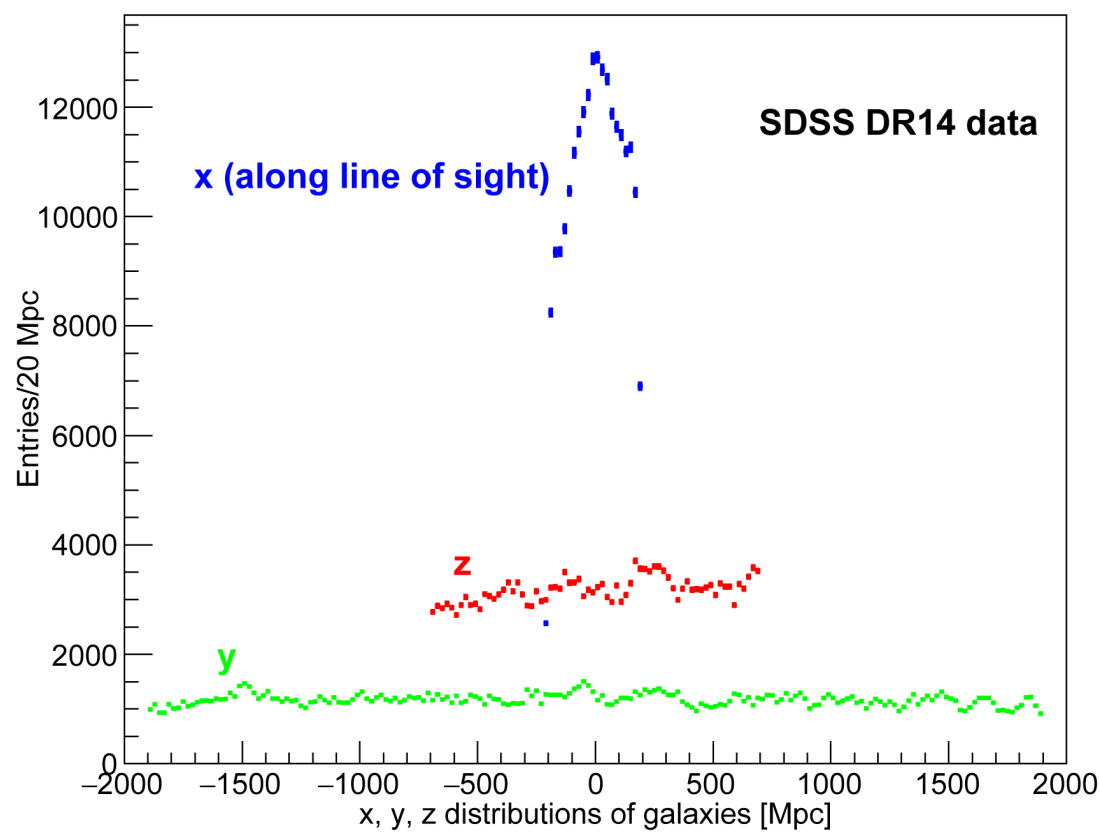

Figure 8. Distributions of 222470 SDSS DR14 galaxies in a "rectangular" box of dimensions $L_{x}=400 \mathrm{Mpc}$ along the line of sight (corresponding to redshift $z=0.5 \pm 0.046$ ), $L_{y}=3800 \mathrm{Mpc}$ (corresponding to an angle $86^{\circ}$ across the sky), and $L_{z}=1400 \mathrm{Mpc}$ (corresponding to an angle $32^{\circ}$ ). 
Some definitions are in order. For data we define the absolute red magnitude of a galaxy MAGr at redshift $z$ as the SDSS DR14 variable -modelMag_r corrected to the reference redshift 0.35 . Similarly, we define the absolute green magnitude of a galaxy MAGg at redshift $z$ as the SDSS DR14 variable -modelMag_g corrected to the reference redshift 0.35 . For a simulated galaxy we define the absolute magnitude MAG $\equiv-19+2.5 \log _{10}\left(M / 10^{16} M_{\odot}\right)$, where $M$ is defined by Equation (24). Note that MAGr and MAGg are derived from observed luminosities, while MAG is derived from the total (baryonic plus dark plus neutrino) mass of the simulation. These quantities can only be compared if the luminosity-to-mass ratio is known.

The number of galaxies per unit volume depends on the limiting magnitude of the survey, or on $I_{\max }$ of the simulation.

\section{Distributions of Galaxies in SDSS DR14 Data and in Simulation}

We would like to obtain $P(k)$ from Equation (19) and Equation (20). Unfortunately we do not have access to the relative density fluctuation $\delta_{c}(\boldsymbol{x}, t)$. Instead we have access to the positions of galaxies and their luminosities. The relation between luminosity and mass of galaxies depends on many variables and is largely unknown, so we focus on the information contained in the positions of galaxies.

Let

$$
n(\boldsymbol{X})=\bar{n}\left(1+\sum_{\boldsymbol{k}^{\prime}} \Delta_{\boldsymbol{k}^{\prime}} \mathrm{e}^{i \boldsymbol{k}^{\prime} \cdot \boldsymbol{X}}\right)
$$

be the number density of point galaxies at redshift $z$ as a function of the comoving coordinate $\boldsymbol{X} \equiv \boldsymbol{x}(t) / a(t)$. We have applied periodic boundary conditions in a comoving volume $V=L_{x} L_{y} L_{z}$, so $\boldsymbol{k}^{\prime}$ has the discrete values of Equation (17). $n(\boldsymbol{X})$ is real, so $\Delta_{-k^{\prime}}=\Delta_{\boldsymbol{k}^{\prime}}^{*}$. The number of galaxies in $V$ is $N_{\text {gal }}=\bar{n} V$. To invert Equation (42), we multiply it by $\exp (-i \boldsymbol{k} \cdot \boldsymbol{X})$, integrate over $V$, and obtain a sum over galaxies $j$ :

$$
\sum_{j} e^{-i k \cdot X_{j}}=N_{g a l} \Delta_{k}+N_{g a l}^{1 / 2} e^{i \varphi} .
$$

The first term on the right hand side of Equation (43) is the result of a coherent sum of $N_{k} \equiv N_{\mathrm{gal}} \Delta_{k}$ terms corresponding to mode $\boldsymbol{k}$. The second term is the result of an incoherent sum which we have approximated to $N_{\mathrm{gal}}^{1 / 2} \mathrm{e}^{i \phi}$, where the phase $\phi$ is arbitrary. We define the "galaxy power spectrum"

$$
P_{\text {gal }}(\boldsymbol{k}) \equiv V\left|\Delta_{k}\right|^{2},
$$

and obtain

$$
P_{\text {gal }}(\boldsymbol{k})=V\left|\frac{1}{N_{\text {gal }}} \sum_{j} \mathrm{e}^{-i \boldsymbol{k} \cdot \boldsymbol{X}_{j}}\right|^{2}-\frac{V}{N_{\text {gal }}} \pm \sqrt{\frac{2 V}{N_{\text {gal }}} P_{\text {gal }}(k) .}
$$

The transition between signal and noise occurs at 
$\log _{10}\left(P_{\text {gal }}(k) / h^{-3} \mathrm{Mpc}^{3}\right) \approx 3.47$ for our data sample, and $\approx 3.49$ for our reference simulation. To test these ideas we can select a narrow range of MAGr, MAGg, or MAG to shift the noise upwards, compare Figures 9-11 (which plot the first term on the right hand side of Equation (45) and include the noise at large $k$ ).

Averaging over $\boldsymbol{k}$ in a bin of $k \equiv|\boldsymbol{k}|$ obtains $P_{\text {gal }}(k)$. The factor $V$ is inserted so that $P_{\text {gal }}(k)$ becomes independent of the arbitrary choice of $V$ for large $V$. The function $P_{\text {gal }}(k)$ defines statistically the distribution of galaxies. The variables $\boldsymbol{k}$ in Equation (16) and Equation (45) should not be confused: there is not necessarily a one-to-one relation between them.

Results for data are presented in Figures 9-11. We note that the galaxy bias $b$ depends on MAGr and MAGg. Even tho $N_{\text {gal }} \gg N_{k}, N_{k}>N_{\text {gal }}^{1 / 2}$ at small $k$. For this reason $P_{\text {gal }}(k)$ in Figure 9 extends to higher $k$ than in Figure 10 and Figure 11 before saturating with noise. Figure 12 presents the noise subtracted galaxy power spectrum $P_{\text {gal }}(k)$, obtained from Figure 9, compared with $P(k)$ calculated with the indicated parameters. Their ratio is the bias $b^{2}$.

Results for the simulations are presented in Figures 13-15. In Figure 15 we compare the reference simulation with $P^{\prime}(k)$, with simulations with $P^{\prime}(k) \cdot\left(-\log _{10}\left(k / h \mathrm{Mpc}^{-1}\right)\right)$ ("steeper slope"), or $P^{\prime}(k) /\left(-\log _{10}\left(k / h \mathrm{Mpc}^{-1}\right)\right)$ ("less slope"). Note that the function $-\log _{10}\left(k / h \mathrm{Mpc}^{-1}\right)$ varies between $\approx 1.3$ to $\approx 0.5$ in the region of interest. We observe, qualitatively, that the slope of $P(k)$ has a larger effect on $P_{\text {gal }}(k)$ than the amplitude $A$. A comparison of the

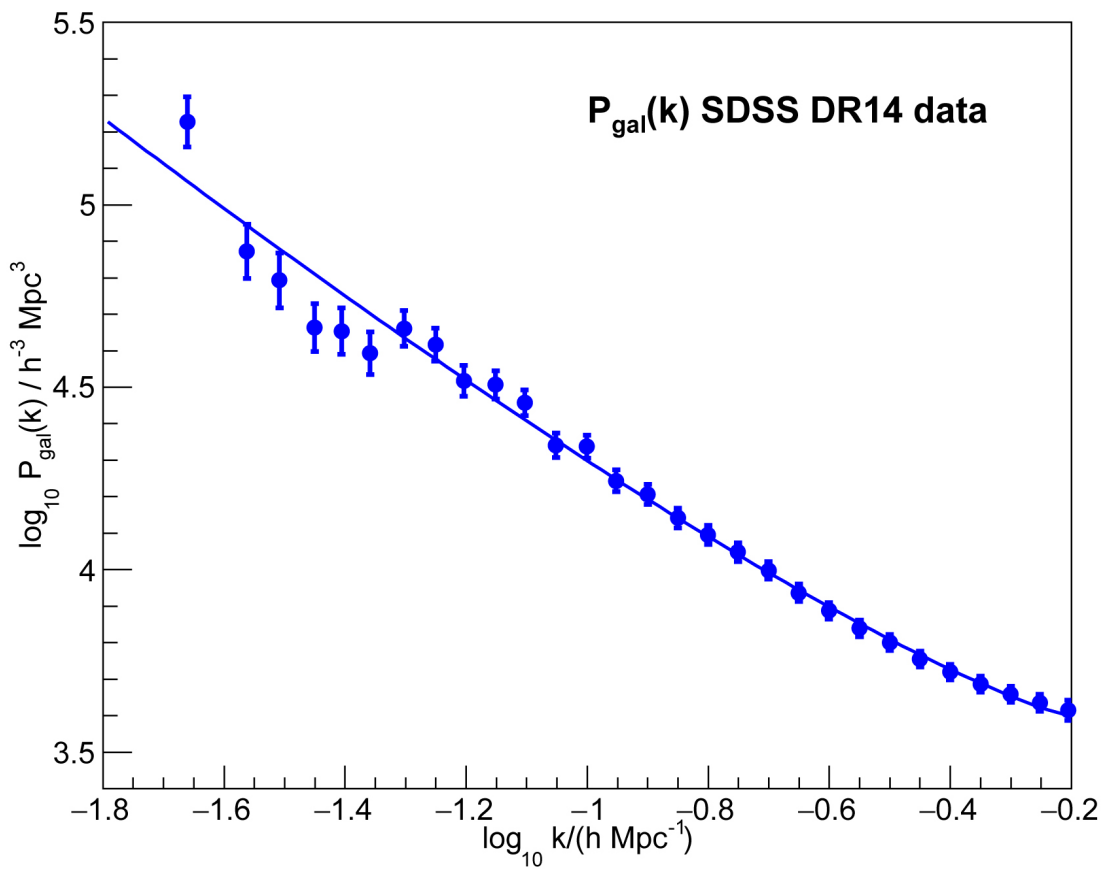

Figure 9. Galaxy power spectrum (plus noise visible at large $k$ ) from SDSS DR14 data in a volume $V=400 \times 3800 \times 1400 \mathrm{Mpc}^{3}$, at redshift $z=0.5 \pm 0.0457$. The fit is $y=3.60+0.92 \cdot(0.2-x)^{1.23}$ with $\chi^{2}=26.8$ for 29 degrees of freedom, where $x$ and $y$ are the axis in this figure. 


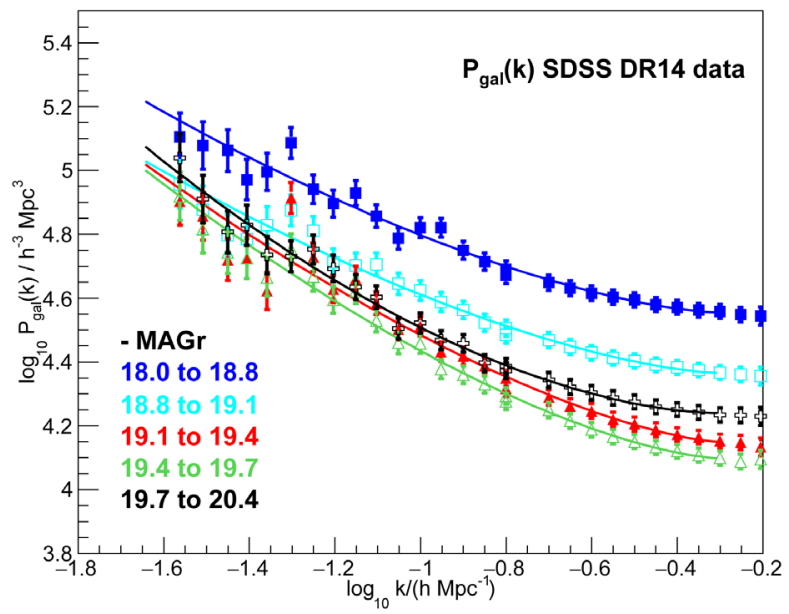

Figure 10. Galaxy power spectrum (plus noise visible at large $k$ ) in bins of MAGr from SDSS DR14 galaxies with redshift $z=0.5 \pm 0.0457$.



Figure 11. Galaxy power spectrum (plus noise visible at large $k$ ) in bins of MAGg from SDSS DR14 galaxies with redshift $z=0.5 \pm 0.0457$.

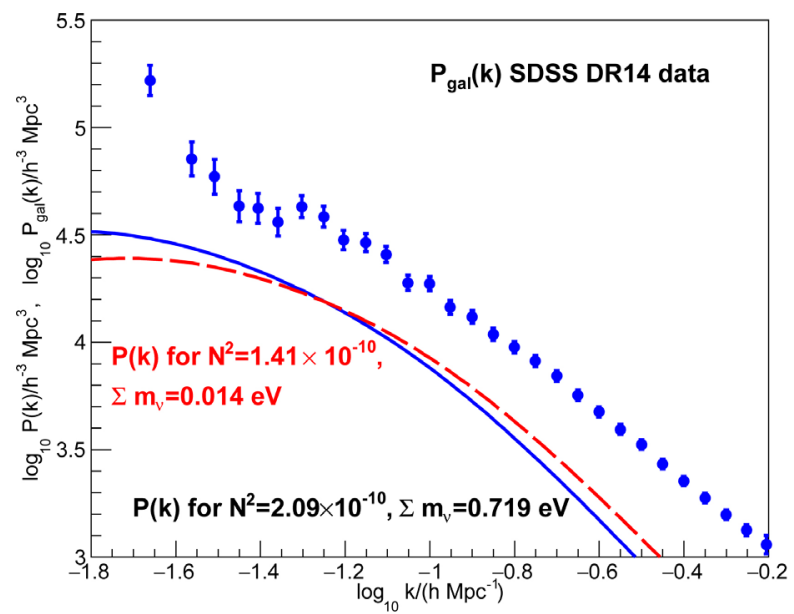

Figure 12. Noise subtracted galaxy power spectrum $P_{\text {gal }}(k)$, obtained from Figure 9, compared with $P(k)$ calculated with the indicated parameters. Their ratio is $b^{2}$. 


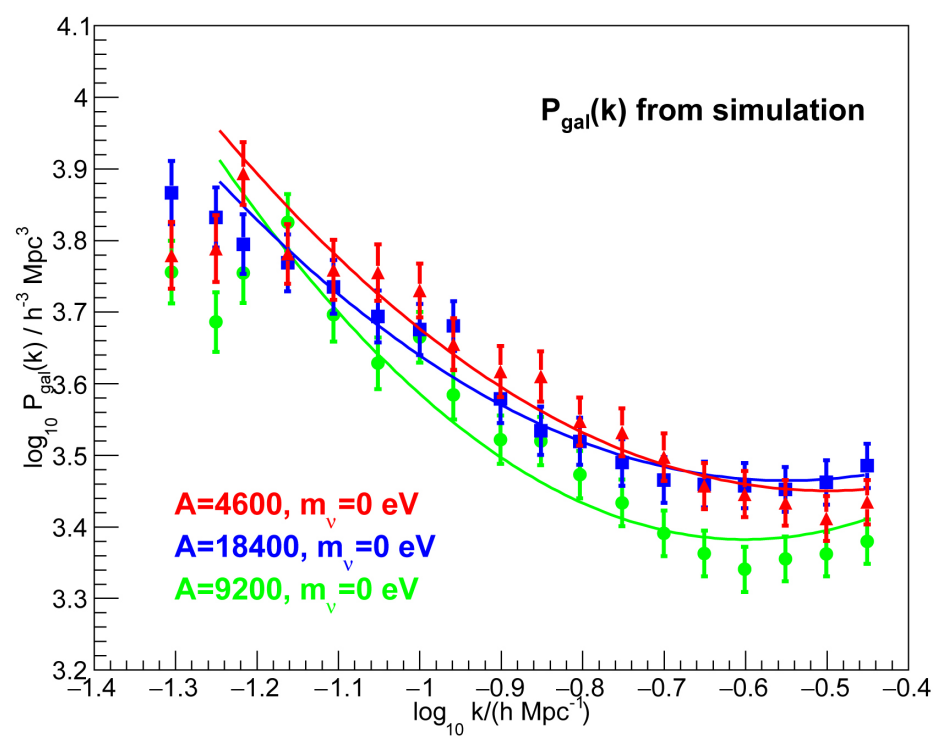

Figure 13. Galaxy power spectrum (plus noise visible at large $k$ ) from simulations with three amplitudes $A$. All other parameters of the simulation are given in Section 5.

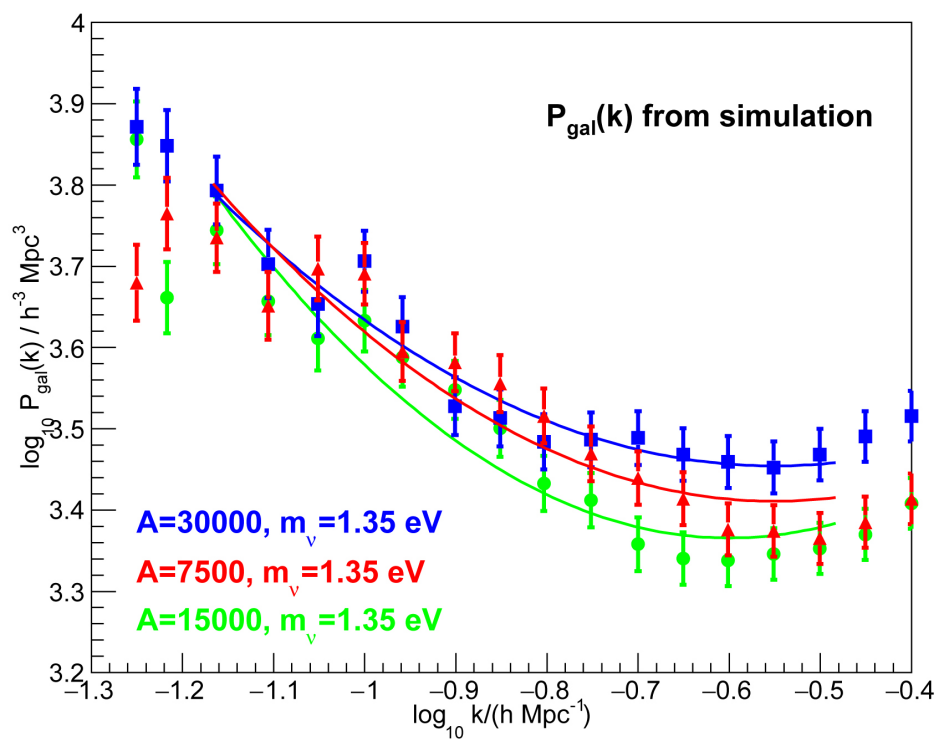

Figure 14. Galaxy power spectrum (plus noise visible at large $k$ ) from simulations with $\sum m_{v}=1.35 \mathrm{eV}$, with three amplitudes $A$. Other parameters are $\eta=4.4$, and $k_{\text {eq }} / h=0.14 \mathrm{Mpc}^{-1}$.

simulations in Figure 15 with $P_{\text {gal }}(k)$ from data in Figure 9 favors a power spectrum $P(k)$ "steeper" than in the reference simulation. The reference simulation has parameters of $P(k)$ similar to the ones obtained from the fit in Figure 1 which assumes scale invariant $b$, and $\sum m_{v}=0 \mathrm{eV}$. The reference simulation is also similar to the fit " $\sum m_{v}=0.014 \mathrm{eV}$ " in Figure 12 (taken from Figure 3 which assumes scale invariant $b$ ). A steeper $P(k)$ implies $\sum m_{v}>0$ as shown in Figure 12 by the curve " $\sum m_{v}=0.719 \mathrm{eV}$ ", and corresponds to a bias $b$ with positive slope as in Equation (55) below. 




Figure 15. Galaxy power spectrum (plus noise visible at large $k$ ) of the reference simulation, a simulation with $P(k)$ with steeper slope $(P(k)$ of Equation (1) is multiplied by $\left.-\log _{10} k /\left(h \mathrm{Mpc}^{-1}\right)\right)$, and a simulation with less slope $(P(k)$ of Equation (1) is divided by $\left.-\log _{10} k /\left(h \mathrm{Mpc}^{-1}\right)\right)$.

\section{Luminosity and Mass Distributions of Galaxies}

Distributions of MAGr and MAGg from data, and MAG from several simulations are presented in Figure 16 and Figure 17. From these figures it is possible to obtain the "mean" luminosity-to-mass ratios. We note that these figures do not show useful sensitivity to $\sum m_{v}$.

\section{Test of Scale Invariance of the Galaxy Bias $b$}

In this Section we test the scale invariance of the bias $b$ defined in Equation (11). To do so, we count galaxies in an array of $N_{s}=N_{x} \times N_{y}$ spheres of radii $r_{s}$, and obtain their mean $\bar{N}$, and their root-mean-square (rms). All spheres have their center at redshift $z=0.5$ to ensure the homogeneity of the galaxy selections. The results for $r_{s}=8 / h, 16 / h, 32 / h, 64 / h, 128 / h$, and $256 / h \mathrm{Mpc}$ are presented in Table 1. The (rms) ${ }^{2}$ has a contribution $\sigma^{2}$ from $P(k)$, and a contribution $\bar{N}$ from statistical fluctuations:

$$
\sigma^{2}=\mathrm{rms}^{2}-\bar{N} \pm 2 \sigma \sqrt{\frac{\bar{N}}{N_{s}}} .
$$

We compare $\sigma / \bar{N}$ obtained from galaxy counts, with the relative mass fluctuations $\sigma_{h r_{s}}$ obtained from Equation (6) and Equation (31). The ratio of these two quantities divided by a correction factor

$$
C\left(\Omega_{\Lambda} /\left(\Omega_{m}(1+z)^{3}\right)\right) /\left(C\left(\Omega_{\Lambda} / \Omega_{m}\right)(1+z)\right)=0.779
$$

[2] is the bias $b$. 




Figure 16. Distributions of MAGr and MAGg of SDSS DR14 data, and distributions of MAG of several simulations (see definitions in Section 5). The difference between the MAGr or MAGg of data and MAG of simulations determines the "mean" galaxy $L / M$ ratio.

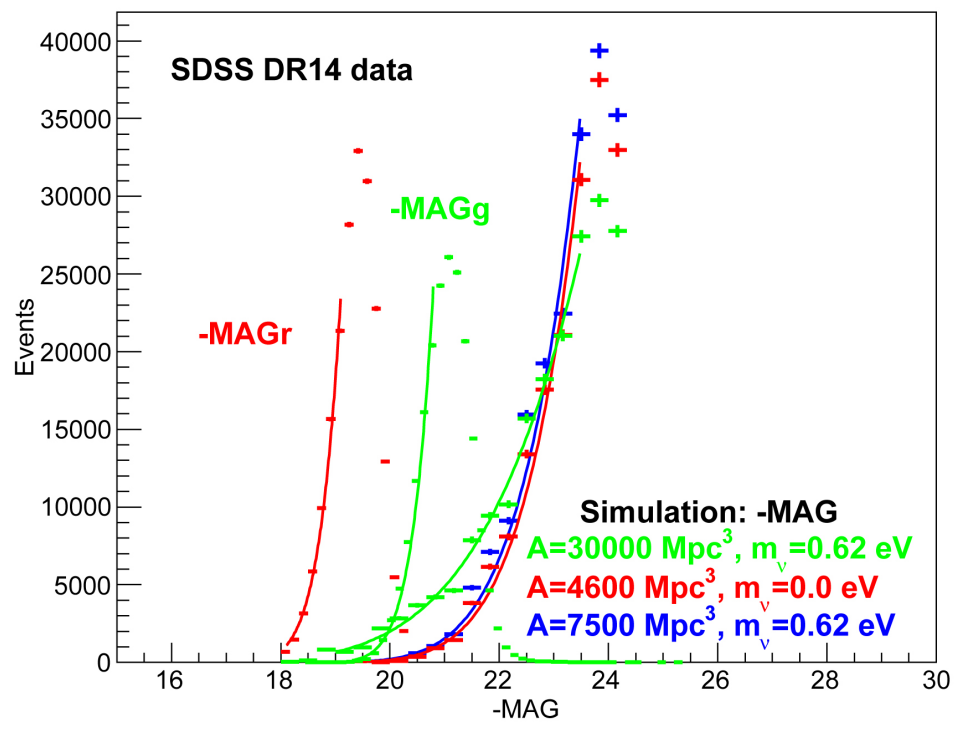

Figure 17. Same as Figure 16 with additional simulations.

The measured bias $b$ is a function of $r_{s}, \sum m_{v}, h$ and the spectral index $n$. Results for $h=0.678$ and $n=1$ are presented in Table 1 . The last column is the $\chi^{2}$ of the five $b$ 's of spheres with $\bar{N}>1$, assuming these $b$ 's are scale invariant with respect to their weighted average. Additional measurements of $\chi^{2}$ are presented in Figure 18. Assuming that $b$ is scale invariant we obtain

$$
\sum m_{v}=0.939+0.035 \cdot \delta h+0.089 \cdot \delta n \pm 0.008 \mathrm{eV},
$$

with minimum $\chi^{2}=3.2$ for four degrees of freedom. We have defined $\delta h \equiv(h-0.678) / 0.009$, and $\delta n \equiv(n-1) / 0.038$. 
Table 1. Mean galaxy counts $\bar{N}$ in spheres of radius $r_{s}$. All spheres have their center at redshift $z=0.5$. The number of spheres is $N_{s}=N_{y} \times N_{z}$. Note that the observed root-mean-square (rms) fluctuation relative to the mean $\bar{N}$ is larger than the corresponding statistical fluctuation, i.e. $\mathrm{rms} / \bar{N}>1 / \sqrt{\bar{N}} \cdot \sigma_{h r_{s}}$ is calculated with Equation (6) and Equation (31) with $N^{2}$ chosen so $\sigma_{8}=0.770$ to set the scale for $b$ (e.g. $N^{2}=0.8472 \times 10^{-10}$ for $\sum m_{v}=0 \mathrm{eV}$, or $N^{2}=1.3575 \times 10^{-10}$ for $\sum m_{v}=0.6 \mathrm{eV}$ ). Both the galaxy counts and $\sigma_{h r_{s}}$ are obtained with the top-hat window function. The true standard deviation is obtained from $\sigma^{2}=\mathrm{rms}^{2}-\bar{N} \pm 2 \sigma \sqrt{\bar{N} / N_{s}}$. The measured "bias" is defined as $b \equiv(\sigma / \bar{N}) /\left(0.779 \cdot \sigma_{h r_{s}}\right)$. The last column is the $\chi^{2}$ of the five $b$ 's of spheres with $\bar{N}>1$, assuming these $b$ 's are scale invariant. $h=0.678, n=1.0$, and $\Omega_{m}=0.281$.

\begin{tabular}{|c|c|c|c|c|c|c|c|}
\hline$h r_{s} \quad[\mathrm{Mpc}]$ & 8 & 16 & 32 & 64 & 128 & 256 & \\
\hline$r_{s}[\mathrm{Mpc}]$ & 11.80 & 23.60 & 47.20 & 94.40 & 188.79 & 377.58 & \\
\hline$N_{y} \times N_{z}$ & $151 \times 55$ & $75 \times 27$ & $37 \times 13$ & $19 \times 7$ & $9 \times 3$ & $4 \times 1$ & \\
\hline $\bar{N}$ & 0.836 & 6.781 & 52.279 & 410.74 & 3092.3 & 21810.0 & \\
\hline $1 / \sqrt{\bar{N}}$ & 1.0935 & 0.3840 & 0.1383 & 0.0493 & 0.0180 & 0.0068 & \\
\hline $\mathrm{rms} / \bar{N}$ & 1.798 & 0.873 & 0.443 & 0.210 & 0.0870 & 0.0346 & \\
\hline$\sigma / \bar{N}$ & $1.427 \pm 0.012$ & $0.784 \pm 0.009$ & $0.421 \pm 0.006$ & $0.204 \pm 0.004$ & $0.085 \pm 0.003$ & $0.034 \pm 0.003$ & \\
\hline$\sigma_{h r_{s}}, \sum m_{v}=0.0 \mathrm{eV}$ & 0.7700 & 0.4457 & 0.2255 & 0.0987 & 0.0374 & 0.0124 & $\chi^{2}$ \\
\hline$b, \sum m_{v}=0.0 \mathrm{eV}$ & $2.380 \pm 0.020$ & $2.257 \pm 0.025$ & $2.398 \pm 0.036$ & $2.650 \pm 0.056$ & $2.925 \pm 0.119$ & $3.503 \pm 0.349$ & 79.2 \\
\hline$\sigma_{h r_{s}}, \sum m_{v}=0.3 \mathrm{eV}$ & 0.7700 & 0.4514 & 0.2321 & 0.1036 & 0.0402 & 0.0136 & \\
\hline$b, \sum m_{v}=0.3 \mathrm{eV}$ & $2.380 \pm 0.020$ & $2.228 \pm 0.024$ & $2.329 \pm 0.035$ & $2.523 \pm 0.053$ & $2.722 \pm 0.111$ & $3.193 \pm 0.318$ & 49.5 \\
\hline$\sigma_{h_{s}}, \sum m_{v}=0.6 \mathrm{eV}$ & 0.7700 & 0.4603 & 0.2425 & 0.1113 & 0.0443 & 0.0152 & \\
\hline$b, \sum m_{v}=0.6 \mathrm{eV}$ & $2.380 \pm 0.020$ & $2.185 \pm 0.024$ & $2.230 \pm 0.033$ & $2.350 \pm 0.049$ & $2.468 \pm 0.100$ & $2.862 \pm 0.285$ & 20.0 \\
\hline$\sigma_{h r_{s}}, \sum m_{v}=0.7 \mathrm{eV}$ & 0.7700 & 0.4640 & 0.2468 & 0.1144 & 0.0460 & 0.0158 & \\
\hline$b, \sum m_{v}=0.7 \mathrm{eV}$ & $2.380 \pm 0.020$ & $2.168 \pm 0.024$ & $2.191 \pm 0.033$ & $2.285 \pm 0.048$ & $2.379 \pm 0.097$ & $2.755 \pm 0.275$ & 12.6 \\
\hline$\sigma_{h r_{s}}, \sum m_{v}=0.8 \mathrm{eV}$ & 0.7700 & 0.4682 & 0.2516 & 0.1179 & 0.0478 & 0.0165 & \\
\hline$b, \sum m_{v}=0.8 \mathrm{eV}$ & $2.380 \pm 0.020$ & $2.148 \pm 0.023$ & $2.149 \pm 0.032$ & $2.218 \pm 0.047$ & $2.289 \pm 0.093$ & $2.648 \pm 0.264$ & 7.1 \\
\hline$\sigma_{h r_{s}}, \sum m_{v}=0.9 \mathrm{eV}$ & 0.7700 & 0.4729 & 0.2570 & 0.1218 & 0.0497 & 0.0171 & \\
\hline$b, \sum m_{v}=0.9 \mathrm{eV}$ & $2.380 \pm 0.020$ & $2.127 \pm 0.023$ & $2.104 \pm 0.032$ & $2.147 \pm 0.045$ & $2.198 \pm 0.089$ & $2.541 \pm 0.253$ & 4.0 \\
\hline$\sigma_{h r_{s}}, \sum m_{v}=1.0 \mathrm{eV}$ & 0.7700 & 0.4782 & 0.2630 & 0.1261 & 0.0519 & 0.0179 & \\
\hline$b, \sum m_{v}=1.0 \mathrm{eV}$ & $2.380 \pm 0.020$ & $2.103 \pm 0.023$ & $2.056 \pm 0.031$ & $2.073 \pm 0.044$ & $2.107 \pm 0.086$ & $2.434 \pm 0.243$ & 3.8 \\
\hline$\sigma_{h r_{s}}, \sum m_{v}=1.2 \mathrm{eV}$ & 0.7700 & 0.4911 & 0.2775 & 0.1363 & 0.0568 & 0.0196 & \\
\hline$b, \sum m_{v}=1.2 \mathrm{eV}$ & $2.380 \pm 0.020$ & $2.048 \pm 0.022$ & $1.948 \pm 0.029$ & $1.919 \pm 0.040$ & $1.923 \pm 0.078$ & $2.220 \pm 0.221$ & 13.7 \\
\hline
\end{tabular}

In conclusion, the galaxy bias $b$ is scale invariant within the statistical uncertainties of $b$ presented in Table 1, provided $\sum m_{v}$ satisfies Equation (47), else scale invariance is broken. Note in Table 1 that the variation of $b$ with scale depends on $\sum m_{v}$.

\section{Measurement of Neutrino Masses with the Sachs-Wolfe Effect and $\sigma_{8}$}

We return to the measurement of neutrino masses. Since the galaxy bias $b$ may be scale dependent, in this Section we exclude measurements of $P_{\text {gal }}(k)$ with galaxies. 


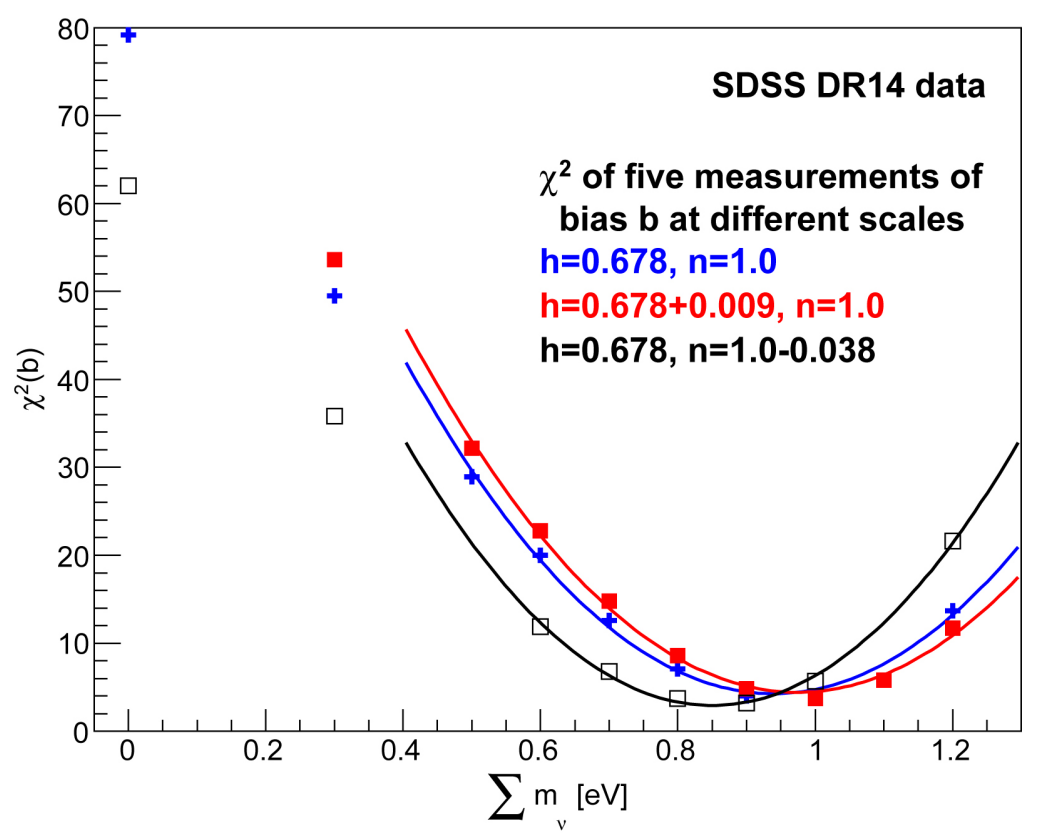

Figure 18. $\chi^{2}$ of five measurements of bias $b$ assumed to be scale invariant with respect to their weighted mean as a function of $\sum m_{v}$, for several values of the Hubble parameter $h$, and the spectral index $n$. The five measurements of $b$ correspond to scales $r_{s}=16 / h, 32 / h, 64 / h, 128 / h$, and $256 / h \mathrm{Mpc}$.

The $\Lambda \mathrm{CDM}$ model is described by Equation (6) that has three free parameters: $N^{2}, n$, and $\sum m_{v}$. We keep $n$ fixed. We vary the two parameters $N^{2}$ and $\sum m_{v}$ to minimize a $\chi^{2}$ with two terms corresponding to two observables: the Sachs-Wolfe effect ( $N^{2}$ from Equation (41)), and $\sigma_{8}$ given by Equation (33). We therefore have zero degrees of freedom. The result is a function of $h, \Omega_{m}$, and the spectral index $n$, so we define $\delta h \equiv(h-0.678) / 0.009 \quad[3]$, $\delta \Omega_{m} \equiv\left(\Omega_{m}-0.281\right) / 0.003 \quad$ [7], and $\delta n \equiv(n-1) / 0.038 \quad$ [3], and obtain

$$
\begin{aligned}
\sum m_{v}= & 0.595+0.047 \cdot \delta h+0.226 \cdot \delta n+0.022 \cdot \delta \Omega_{m} \\
& \pm 0.225(\text { stat })_{-0.152}^{+0.484}(\mathrm{syst}) \mathrm{eV} .
\end{aligned}
$$

Note that in the "6 parameter $\Lambda \mathrm{CDM}$ fit" [3], which assumes $\sum m_{v}=0.06$ $\mathrm{eV}, n=0.968 \pm 0.006$. Here, and below, the systematic uncertainties are obtained by repeating the fits with the top-hat window function instead of the gaussian window function for $\sigma_{8}$ (and for $\sigma / \bar{N}$ if applicable), and also with $\sigma_{8}=0.815 \pm 0.009$ obtained with the "6 parameter $\Lambda$ CDM fit" [3], instead of $\sigma_{8}$ from direct measurements, Equation (33).

The fit of Equation (48) is compared with measurements of $P_{\text {gal }}(k)$ obtained from the SDSS-III BOSS survey [4] in Figure 19. It is interesting to note that the discrepancy, i.e. the drop of $P(k)$ in the range $-1.6<\log _{10}(k / h \mathrm{Mpc})<-1.3$, is also observed in Figure 12.

For comparison, reference [8] obtains $\Omega_{m}=0.282 \pm 0.003$ and

$$
\sum m_{v}=0.711-0.335 \cdot \delta h+0.050 \cdot \delta b \pm 0.063 \mathrm{eV},
$$




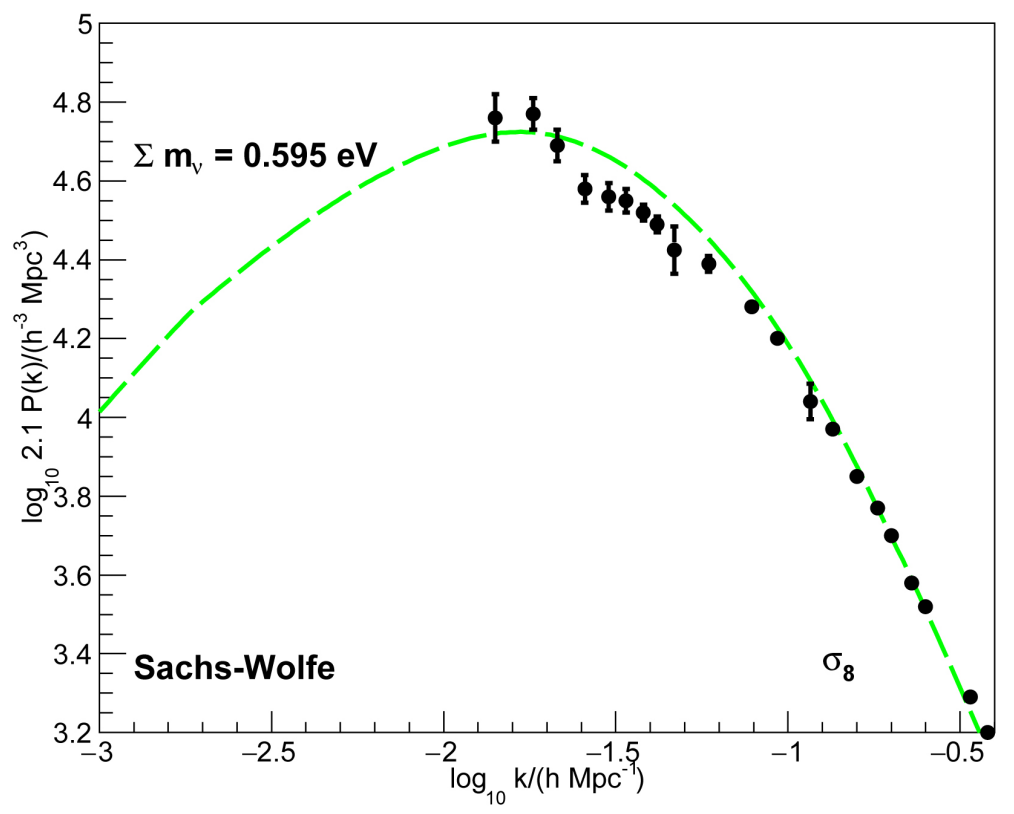

Figure 19. Comparison of $P_{\text {gal }}(k)$ obtained from the SDSS-III BOSS survey [4] ("reconstructed") with $b^{2} P(k)$ obtained from a fit of Equation (6) to the Sachs-Wolfe effect and $\sigma_{8}$ only. The fit obtains $\sum m_{v}=0.595 \pm 0.225 \mathrm{eV}$ with zero degrees of freedom. $h=0.678$ and $n=1.0$ are fixed.

where $\delta b \equiv\left(\Omega_{b} h^{2}-0.02226\right) / 0.00023$, from a study of BAO with SDSS DR13 galaxies. We allow $\Omega_{b} h^{2}$ to vary by one standard deviation, i.e. $\delta b=0 \pm 1$ [7]. To combine the independent measurements (48) and (49) we add one more term to the $\chi^{2}$ corresponding to the measurement (49), so we now have one degree of freedom. We obtain

$$
\begin{aligned}
\sum m_{v}= & 0.696-0.281 \cdot \delta h+0.032 \cdot \delta n+0.003 \cdot \delta \Omega_{m} \\
& \pm 0.075(\text { stat })_{-0.029}^{+0.055}(\text { syst }) \mathrm{eV}
\end{aligned}
$$

with $\chi^{2}=0.25$ for one degree of freedom, so the two independent measurements of $\sum m_{v}$, Equation (48) and Equation (49), are consistent. Note that the uncertainty of $h$ dominates the uncertainty of $\sum m_{v}$ in Equation (50).

We now free $h$ and add one term to the $\chi^{2}$ corresponding to $h=0.0678 \pm 0.009$ [3], and obtain

$$
\begin{gathered}
\sum m_{v}=0.633 \pm 0.168(\text { stat })_{-0.043}^{+0.064}(\text { syst }) \mathrm{eV} \\
h=0.680 \pm 0.005(\text { stat })
\end{gathered}
$$

with $\chi^{2}=0.07$ for one degree of freedom. The systematic uncertainties in Equation (51) now include $\delta n$. The $1 \sigma, 2 \sigma$, and $3 \sigma$ contours are presented in Figure 20.

If instead we set $h=0.72 \pm 0.03$ from the direct measurement of the Hubble expansion rate [3], we obtain

$$
\sum m_{v}=0.563 \pm 0.207(\text { stat })_{-0.043}^{+0.064}(\text { syst }) \mathrm{eV}
$$




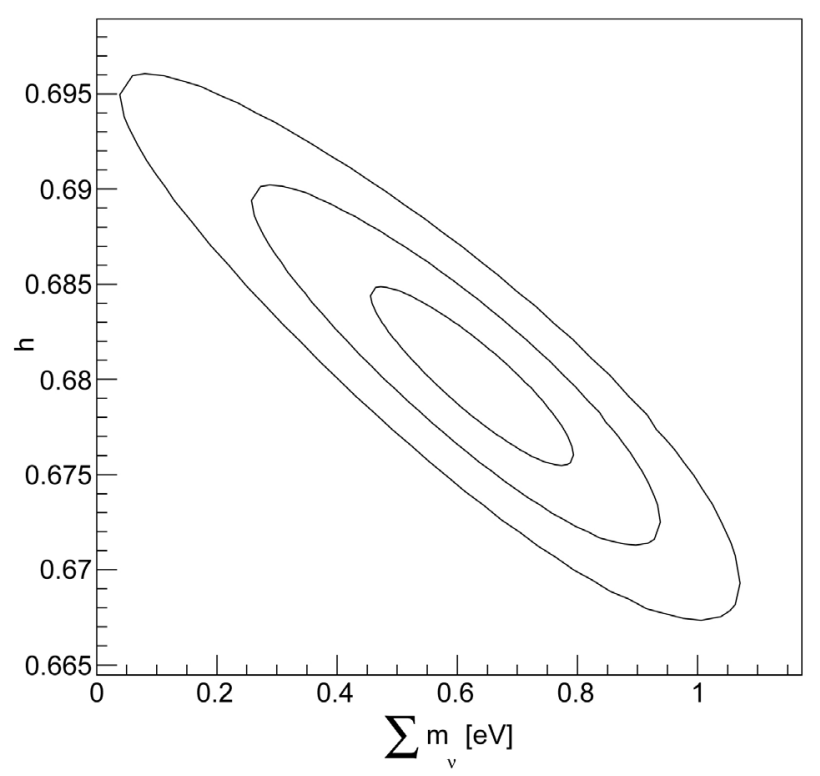

Figure 20. Contours corresponding to 1,2 , and 3 standard deviations in the $\left(\sum m_{v}, h\right)$ plane, from Sachs-Wolfe, $\sigma_{8}, h=0.678 \pm 0.009$, and BAO measurements. Points on the contours have $\chi^{2}-\chi_{\min }^{2}=1,4$, and 9 , respectively, where $\chi^{2}$ has been minimized with respect to $N^{2}$. The total uncertainty of $\sum m_{v}$ is dominated by the uncertainty of $h$. In this figure $n=1$, and the systematic uncertainties, presented in Equation (51), are not included.

$$
h=0.682 \pm 0.006(\text { stat }) \text {, }
$$

with $\chi^{2}=1.7$ for 1 degree of freedom. The corresponding $1 \sigma, 2 \sigma$, and $3 \sigma$ contours are presented in Figure 21. Note that the fitted $h$ does not change significantly.

\section{Measurement of Neutrino Masses with the Sachs-Wolfe Effect, $\sigma_{8}$, and $P_{\text {gal }}(k)$}

We repeat the fit of Figure 3, which includes the "reconstructed" SDSS-III BOSS $P_{\text {gal }}(k)$ measurements [4], but this time we allow the galaxy bias $b$ to depend on scale: $b \equiv b_{0}+b_{1} \log _{10}\left(k / h \mathrm{Mpc}^{-1}\right)$. Minimizing the $\chi^{2}$ with respect to $\sum m_{v}$, $N^{2}, n, h=0.678 \pm 0.009, b_{0}$, and $b_{1}$, we obtain

$$
\begin{gathered}
\sum m_{v}=0.80 \pm 0.23 \mathrm{eV}, \\
N^{2}=(1.88 \pm 0.39) \times 10^{-10}, \\
n=1.064 \pm 0.068, \\
h=0.676 \pm 0.011, \\
b_{0}=2.35 \pm 0.36, \\
b_{1}=0.229 \pm 0.094,
\end{gathered}
$$

with $\chi^{2}=27.8$ for 18 degrees of freedom. The uncertainties have been multiplied by $\sqrt{27.8 / 18}$. Confidence contours are presented in Figure 22. Fixing 


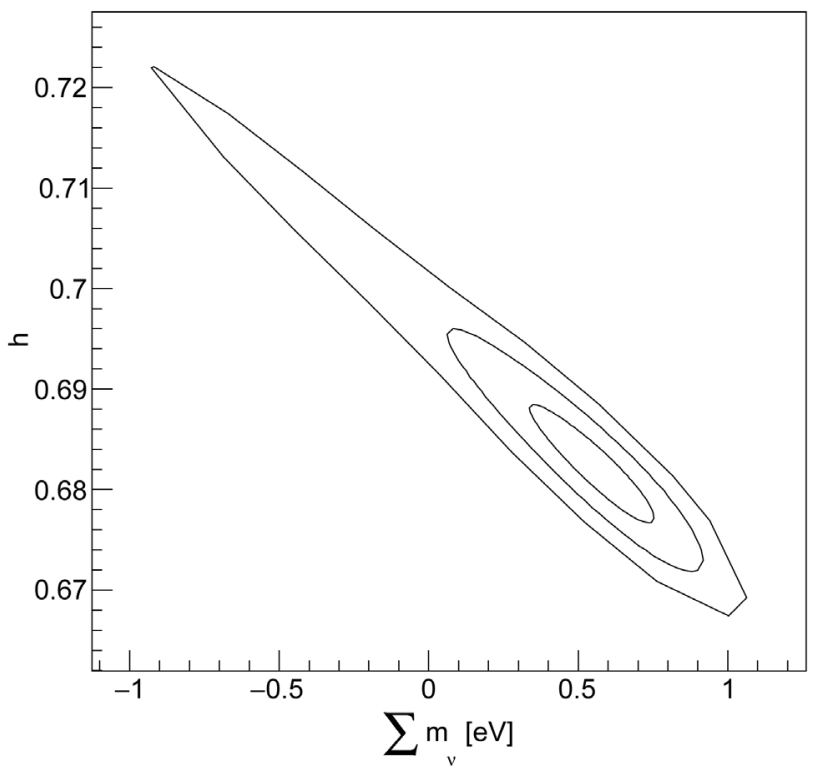

Figure 21. Same as Figure 20 but $h=0.72 \pm 0.03$.

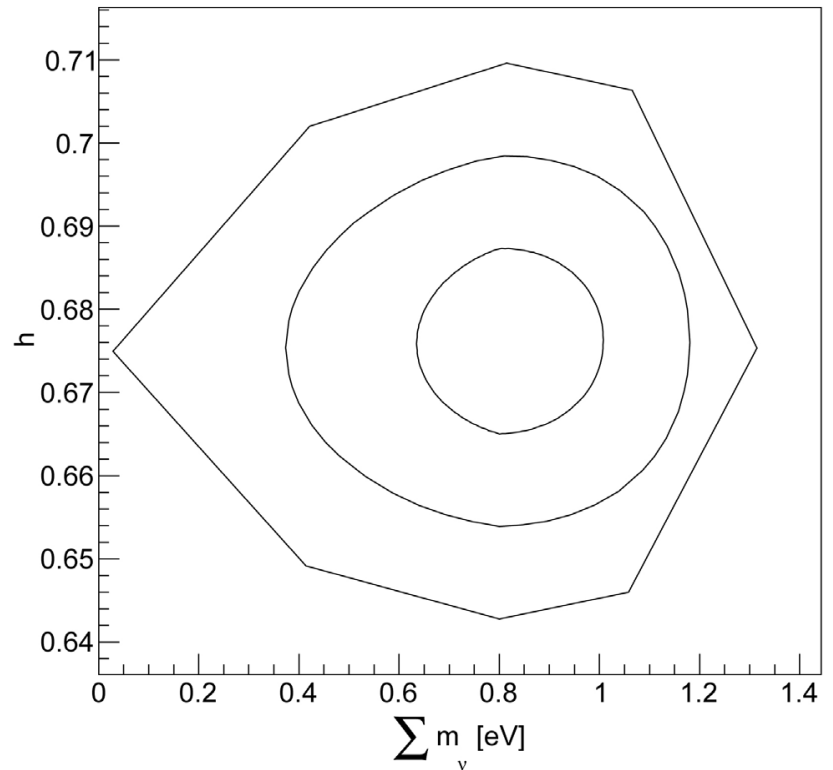

Figure 22. Contours corresponding to 1,2 , and 3 standard deviations in the $\left(\sum m_{v}, h\right)$ plane, from Sachs-Wolfe, $\sigma_{8}, h=0.678 \pm 0.009$, and $P_{\text {gal }}(k)$ measurements. Points on the contours have $\chi^{2}-\chi_{\min }^{2}=1,4$, and 9 , respectively, where $\chi^{2}$ has been minimized with respect to $N^{2}, n, b_{0}$, and $b_{1}$.

$b_{1}=0$ obtains $\chi^{2}=36.3$, so including the scale dependence of $b$ is necessary.

\section{Measurement of Neutrino Masses with the Sachs-Wolfe Effect, $\sigma_{8}$, and Galaxy Fluctuations}

We repeat the measurements of $\sum m_{v}$ of Section 9 but add 4 more experimental constraints: $\sigma / \bar{N}$ of galaxy counts in spheres of radius 
$r_{s}=16 / h, 32 / h, 64 / h$, and $128 / h \mathrm{Mpc}$, which are listed in Table 1 . Spheres of radius $8 / h \mathrm{Mpc}$ were not considered because they have $\bar{N}<1$. Spheres of radius $256 / h \mathrm{Mpc}$ were excluded because there are only 4 spheres of this radius, and the difference between the rms for the top-hat and gaussian window functions turns out to be large (while consistent results are obtained for the other radii). We add two more parameters to be fit: $b_{0}$ and $b_{s}$ which define the bias $b=b_{0}-i_{s} b_{s}$, with $i_{s}=0,1,2,3$ for $r_{s}=16 / h, 32 / h, 64 / h$, and $128 / h \mathrm{Mpc}$, respectively. Note that we do not obtain a good fit with fixed bias $b=b_{0}$, and so have introduced a "bias slope" $b_{s}$.

From the Sachs-Wolfe effect, $\sigma_{8}$, and the $4 \sigma / \bar{N}$ measurements we obtain

$$
\begin{aligned}
\sum m_{v}= & 0.618+0.042 \cdot \delta h+0.206 \cdot \delta n+0.019 \cdot \delta \Omega_{m} \\
& \pm 0.209(\text { stat })_{-0.139}^{+0.420}(\mathrm{syst}) \mathrm{eV}
\end{aligned}
$$

with $\chi^{2}=1.10$ for 2 degrees of freedom. The variables that minimize the $\chi^{2}$ are $\sum m_{v}, N^{2}, b_{0}$, and $b_{s}$. This result may be compared with (48).

Freeing $h=0.678 \pm 0.009$, and keeping $n=1.0$ fixed, we obtain

$$
\begin{gathered}
\sum m_{v}=0.618 \pm 0.214 \mathrm{eV}, \\
N^{2}=(2.11 \pm 0.31) \times 10^{-10}, \\
h=0.678 \pm 0.009, \\
b_{0}=1.756 \pm 0.057, \\
b_{s}=-0.062 \pm 0.042,
\end{gathered}
$$

with $\chi^{2}=1.10$ for 2 degrees of freedom.

Combining with the BAO measurement (49) we obtain

$$
\begin{aligned}
\sum m_{v}= & 0.697-0.276 \cdot \delta h+0.032 \cdot \delta n+0.003 \cdot \delta \Omega_{m} \\
& \pm 0.075(\text { stat })_{-0.028}^{+0.055}(\mathrm{syst}) \mathrm{eV},
\end{aligned}
$$

with $\chi^{2}=1.29$ for 3 degrees of freedom. The variables that minimize the $\chi^{2}$ are $\sum m_{v}, N^{2}, b_{0}$, and $b_{s}$. Freeing $h=0.678 \pm 0.009$, and keeping $n=1.0$ fixed, we obtain

$$
\begin{gathered}
\sum m_{v}=0.644 \pm 0.162 \mathrm{eV}, \\
N^{2}=(2.13 \pm 0.28) \times 10^{-10}, \\
h=0.680 \pm 0.005, \\
b_{0}=1.756 \pm 0.057, \\
b_{s}=-0.058 \pm 0.036,
\end{gathered}
$$

with $\chi^{2}=1.14$ for 3 degrees of freedom.

Finally, freeing $n$, and minimizing the $\chi^{2}$ with respect to $\sum m_{v}, N^{2}, n$, $h=0.678 \pm 0.009, b_{0}$, and $b_{s}$, we obtain

$$
\sum m_{v}=0.719 \pm 0.312(\text { stat })_{-0.028}^{+0.055}(\text { syst }) \mathrm{eV},
$$




$$
\begin{gathered}
N^{2}=(2.09 \pm 0.33) \times 10^{-10}, \\
n=1.021 \pm 0.075, \\
h=0.678 \pm 0.008, \\
b_{0}=1.751 \pm 0.060, \\
b_{s}=-0.053 \pm 0.041,
\end{gathered}
$$

with $\chi^{2}=1.1$ for 2 degrees of freedom. The parameter correlation coefficients, defined in [3], are

\begin{tabular}{ccccccc}
\hline & $\sum m_{v}$ & $N^{2}$ & $n$ & $h$ & $b_{0}$ & $b_{s}$ \\
\hline$\sum m_{v}$ & 1.000 & -0.019 & 0.856 & -0.966 & -0.226 & 0.779 \\
$N^{2}$ & -0.019 & 1.000 & -0.491 & 0.018 & -0.155 & 0.428 \\
$n$ & 0.856 & -0.491 & 1.000 & -0.834 & -0.303 & 0.427 \\
$h$ & -0.966 & 0.018 & -0.834 & 1.000 & 0.219 & -0.755 \\
$b_{0}$ & -0.226 & -0.155 & -0.303 & 0.219 & 1.000 & -0.037 \\
$b_{s}$ & 0.779 & 0.428 & 0.427 & -0.755 & -0.037 & 1.000 \\
\hline
\end{tabular}

Note that we have measured the amplitude $N^{2}$ and spectral index $n$ of $P(k)$, and the bias $b_{0}$ including its slope $b_{s}$ for the SDSS DR14 galaxy selections at redshift $z=0.5 .1,2,3$, and 4 standard deviation contours are presented in Figure 23.

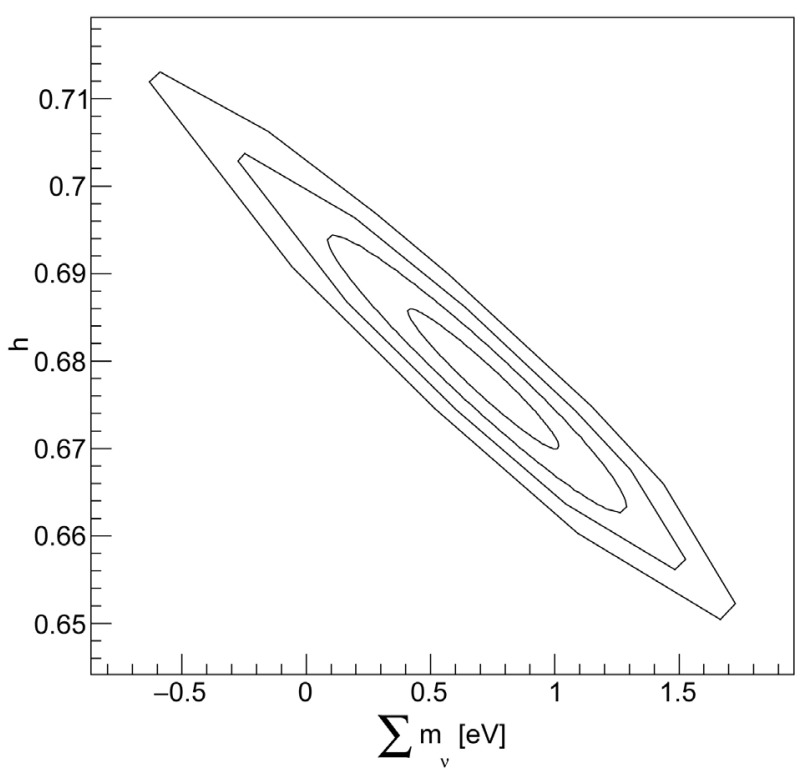

Figure 23. Contours corresponding to $1,2,3$, and 4 standard deviations in the $\left(\sum m_{v}, h\right)$ plane, from Sachs-Wolfe, $\sigma_{8}, 4 \sigma / \bar{N}, \mathrm{BAO}$, and $h=0.678 \pm 0.009$ measurements. Points on the contours have $\chi^{2}-\chi_{\min }^{2}=1,4,9$, and 16, respectively, where $\chi^{2}$ has been minimized with respect to $N^{2}, n, b_{0}$, and $b_{s}$. The total uncertainty of $\sum m_{v}$ is dominated by the uncertainty of $h$. In this figure the systematic uncertainties, presented in Equation (60), are not included. 
Figure 23 and Equation (60) are our final results.

\section{Conclusions}

We have studied galaxy distributions with Sloan Digital Sky Survey SDSS DR14 data and with simulations searching for variables that can constrain neutrino masses. Fitting the predictions of the $\Lambda \mathrm{CDM}$ model to the Sachs-Wolfe effect, $\sigma_{8}, P_{\text {gal }}(k)$, fluctuations of galaxy counts in spheres of radii ranging from $16 / \mathrm{h}$ to $128 / h \mathrm{Mpc}, \mathrm{BAO}$ measurements, and $h=0.678 \pm 0.009$, in various combinations, with free spectral index $n$, and free galaxy bias and galaxy bias slope, we obtain consistent measurements of $\sum m_{v}$. The uncertainty of $\sum m_{v}$ is dominated by the uncertainty of $h$, so we have presented confidence contours in the $\left(\sum m_{v}, h\right)$ plane.

Fitting the predictions of the $\Lambda \mathrm{CDM}$ model to the Sachs-Wolfe effect and $\sigma_{8}$ we obtain (48). Fitting the predictions of the $\Lambda$ CDM model to the Sachs-Wolfe effect, $\sigma_{8}$, and galaxy number fluctuations $\sigma / \bar{N}$ in spheres of radius $r_{s}=16 / h, 32 / h, 64 / h$, and $128 / h$, we obtain (56). These results are consistent with the measurement (49) with BAO. Combining these last two independent measurements we obtain

$$
\begin{aligned}
\sum m_{v}= & 0.697-0.276 \cdot \delta h+0.032 \cdot \delta n+0.003 \cdot \delta \Omega_{m} \\
& \pm 0.075(\text { stat })_{-0.028}^{+0.055}(\text { syst }) \mathrm{eV} .
\end{aligned}
$$

Note that the uncertainty of $\sum m_{v}$ is dominated by the uncertainty of $h$. A global fit with $h=0.678 \pm 0.009$ obtains $\sum m_{v}=0.719 \pm 0.312$ (stat $)_{-0.028}^{+0.055}$ (syst) $\mathrm{eV}, h=0.678 \pm 0.008$, and the amplitude and spectral index of $P(k)$ : $N^{2}=(2.09 \pm 0.33) \times 10^{-10}$, and $n=1.021 \pm 0.075$. The fit also returns the galaxy bias $b$ including its scale dependence.

Figure 23 and Equation (60) are our final results. These results follow from the data analyzed and the assumptions of the validity of the $\Lambda \mathrm{CDM}$ model and $h=0.678 \pm 0.009$. The measured $\sum m_{v}$ is anticorrelated with $h$. All steps in this analysis have been fully described.

Note added in proof: Let us comment on Equations (49) and (56). Equation (49) is mainly determined by the precise measurement of the sound horizon angle $\theta_{M C}$ by the Planck experiment, and by the assumption that the BAO wave stalls at redshift $z=z_{*}=1089.9 \pm 0.4$. Equation (49) tells us that $\left(\sum m_{v}, h\right)$ lies on the diagonal shown in Figure 23 (with some uncertainty from $\Omega_{b} h^{2}$ ). Equation (56) is a constraint mainly between $\sum m_{v}$ and $n$ with large uncertainties. To determine $\sum m_{v}$ we need as input a value for $h$ (or a value for $n$ ). In this article we have taken $h=0.678 \pm 0.009$ from [3]. If $h=0.678 \pm 0.009$ we obtain $\sum m_{v}=0.719 \pm 0.312 \mathrm{eV}$, and $n=1.021 \pm 0.075$. If however $h=0.688 \pm 0.009$ we obtain $\sum m_{v}=0.412 \pm 0.328 \mathrm{eV}$, and $n=0.960 \pm 0.073$. And if $h \approx 0.697$, we obtain $\sum m_{v} \approx 0 \mathrm{eV}$. Alternatively, if we fix $n=1.0$, then $h=0.681 \pm 0.005$ and $\sum m_{v}=0.619 \pm 0.182 \mathrm{eV}$. Or if we fix $n=0.968$ [3], then $h=0.684 \pm 0.005$ and $\sum m_{v}=0.486 \pm 0.183 \mathrm{eV}$. At the Guadeloupe 2018 
Conference, Adam Riess, representing the $\mathrm{SH}_{0} \mathrm{ES}$ Team, presented the latest direct measurement of the expansion parameter: $h=0.7353 \pm 0.0162$, which corresponds to negative $\sum m_{v}$ ! The solution may come from an unexpected direction: gravitational waves from merging black holes are a "standard siren". The single black hole merger GW 170817 already obtains $h=0.70_{-0.08}^{+0.12}$, see the talk by Archil Kobakhidze!

\section{Acknowledgements}

Funding for the Sloan Digital Sky Survey (SDSS) has been provided by the Alfred P. Sloan Foundation, the Participating Institutions, the National Aeronautics and Space Administration, the National Science Foundation, the U.S. Department of Energy, the Japanese Monbukagakusho, and the Max Planck Society. The SDSS Web site is http://www.sdss.org/.

The SDSS is managed by the Astrophysical Research Consortium (ARC) for the Participating Institutions. The Participating Institutions are The University of Chicago, Fermilab, the Institute for Advanced Study, the Japan Participation Group, The Johns Hopkins University, Los Alamos National Laboratory, the Max-Planck-Institute for Astronomy (MPIA), the Max-Planck-Institute for Astrophysics (MPA), New Mexico State University, University of Pittsburgh, Princeton University, the United States Naval Observatory, and the University of Washington.

\section{Conflicts of Interest}

The authors declare no conflicts of interest regarding the publication of this paper.

\section{References}

[1] Sachs, R.K. and Wolfe, A.M. (1967) Perturbations of a Cosmological Model and Angular Variations of the Microwave Background. The Astrophysical Journal, 147, 73. https://doi.org/10.1086/148982

[2] Steven Weinberg (2008) Cosmology. Oxford University Press, New York.

[3] Patrignani, C., et al. (2016) Review of Particle Physics. Chinese Physics C, 40, 100001. https://doi.org/10.1088/1674-1137/40/10/100001

[4] Anderson, L., et al. (2012) The Clustering of Galaxies in the SDSS-III Baryon Oscillation Spectroscopic Survey: Baryon Acoustic Oscillations in the Data Release 9 Spectroscopic Galaxy Sample. MNRAS, 427, 3435. https://doi.org/10.1111/j.1365-2966.2012.22066.x

[5] Blanton, M.R., et al. (2017) Sloan Digital Sky Survey IV: Mapping the Milky Way, Nearby Galaxies, and the Distant Universe. The Astronomical Journal, 154, Article ID: 28.

[6] Dawson, K.S., et al. (2013) The Baryon Oscillation Spectroscopic Survey of SDSS-III. The Astronomical Journal, 145, Article ID: 10.

[7] Hoeneisen, B. (2017) Study of Baryon Acoustic Oscillations with SDSS DR13 Data and Measurements of $\Omega_{k}$ and $\Omega_{\mathrm{DE}}(a)$. International Journal of Astronomy and 
Astrophysics, 7, 11-27. https://doi.org/10.4236/ijaa.2017.71002

[8] Hoeneisen, B. (2018) Constraints on Neutrino Masses from Baryon Acoustic Oscillation Measurements. International Journal of Astronomy and Astrophysics, 8, 1-5. https://doi.org/10.4236/ijaa.2018.81001

[9] Lesgourgues J. and Pastor S. (2006) Massive Neutrinos and Cosmology. Physics Reports, 429, 307. https://doi.org/10.1016/j.physrep.2006.04.001

[10] Feldman H.A., Kaiser N. and Peacock J.A. (1994) Power-Spectrum Analysis of Three-Dimensional Redshift Surveys. ApJ, 426, 23. https://doi.org/10.1086/174036

[11] Peebles, P.J.E. (1980) The Large-Scale Structure of the Universe. Princeton University Press.

[12] Hoeneisen, B. (2000) A Simple Model of the Hierarchical Formation of Galaxies. arXiv:astro-ph/0009071 